\title{
On the existence and stability of two positive solutions of a hybrid differential system of arbitrary fractional order via Avery-Anderson-Henderson criterion on cones
}

\author{
Mohammed M. Matar ${ }^{1}$, Manar abu Jarad, Manzoor Ahmad², Akbar Zada²*, Sina Etemad³ and \\ Shahram Rezapour ${ }^{3,4^{*}}$ (i)
}

${ }^{*}$ Correspondence:

akbarzada@uop.edu.pk:

sh.rezapour@azaruniv.ac.ir; sh.rezapour@mail.cmuh.org.tw;

rezapourshahram@yahoo.ca

²Department of Mathematics,

University of Peshawar, Peshawar

25000, Pakistan

${ }^{3}$ Department of Mathematics,

Azarbaijan Shahid Madani

University, Tabriz, Iran

Full list of author information is

available at the end of the article

\section{Springer}

\begin{abstract}
The main objective of this paper is to investigate the existence, uniqueness, and Ulam-Hyers stability of positive solutions for fractional integro-differential boundary values problem. Uniqueness result is obtained by using the Banach principle. For obtaining two positive solutions, we apply another fixed point criterion due to Avery-Anderson-Henderson on cones by establishing some inequalities. An illustrative example is presented to indicate the validity of the obtained results. The results are new and provide a generalization to some known results in the literature.
\end{abstract}

MSC: Primary 34A08; secondary 34A12

Keywords: Boundary values problem; Fixed point; Fractional derivative; Ulam-Hyers stability

\section{Introduction}

The topic of fractional boundary values problems gained a largest share of interest of researchers and scientists due to its great and important role in many fields such as engineering, physics, chemistry, and many other applications, see [1-3] and the references therein.

The subject of analysis of differential systems such as existence, uniqueness, and stability of solution for various boundary values problems has received the attention of many researchers, since the shape of the solution of differential models is obtained by its boundary [4-15]. One form of active research is the hybrid system that has been used as a model of several physical systems and has an unusual differential form, see [16-23].

The fixed point theorems of many versions are the main core of obtaining the necessary and sufficient criteria implying the existence and uniqueness of solution for fractional boundary values problems [24-42]. In particular, Banach fixed point theorem is the most

(c) The Author(s) 2021. This article is licensed under a Creative Commons Attribution 4.0 International License, which permits use, sharing, adaptation, distribution and reproduction in any medium or format, as long as you give appropriate credit to the original author(s) and the source, provide a link to the Creative Commons licence, and indicate if changes were made. The images or other third party material in this article are included in the article's Creative Commons licence, unless indicated otherwise in a credit line to the material. If material is not included in the article's Creative Commons licence and your intended use is not permitted by statutory regulation or exceeds the permitted use, you will need to obtain permission directly from the copyright holder. To view a copy of this licence, visit http://creativecommons.org/licenses/by/4.0/. 
popular one to find the unique solution of the problem. The existence of more than one solution has been obtained by many fixed point theorems such as Schauder's and Krasnosel'skii's fixed point theorems according to the stated given conditions on nonlinear terms. The existence of at least two solutions of the nonlinear boundary value problem (BVP) are given by Avery-Anderson-Henderson fixed point principle [43].

The in-depth qualitative behavior of the solution for fractional BVPs is the positivity of such solutions. The study of existence and stability of positive solution in boundary value problems is characterized by more investigation in all components of the fractional models along with the involved boundary conditions [44, 45]. Most researchers avoid the multi nonzero components in initial or boundary conditions such as constants, functions, integrals, or even derivatives of functions. The using of the zero-valued-conditions fasciate these investigations and avoid any conflicts of the components.

Sun et al. [46] investigated the required conditions for confirming the existence and uniqueness of the solution to a nonlinear fractional differential equation (FDE) whose nonlinearity involves an explicit fractional derivative using Avery-Anderson-Henderson fixed point theorem. Devi et al. [44] studied the existence and uniqueness along with the Ulam-Hyers (UH) stability of positive solution of general nonlinear FDEs containing $p$-Laplacian operator. The authors of [47] turned to the existence and multiplicity of positive solutions for a system consisting of Riemann-Liouville FDEs equipped with the p-Laplacian operators and singular nonnegative nonlinearities, and also furnished with nonlocal boundary conditions which possess the integrals of Riemann-Stieltjes type. The existence criterion and its stability of a hybrid fractional differential equation with fractional integral, fractional derivative in the Caputo sense, and $p$-Laplacian operator are also investigated in the research article by Al-Sadi et al. [48].

In this article, we focus on the fractional integro-differential boundary value problem of a hybrid system given as

$$
\left\{\begin{array}{l}
D^{\alpha}(x(t)-g(t, x(t)))=f(t, x(t)), \quad t \in\left(t_{0}, T\right), \alpha \in(n-1, n), \\
m_{0} x\left(t_{0}\right)+n_{0} D^{\rho_{0}} x(T)=I^{\delta_{0}} h_{0}(T, x(T)), \quad \rho_{0} \in(0,1), m_{0}, n_{0} \in \mathbb{R} \\
m_{1} x\left(t_{0}\right)+n_{1} D^{\rho_{1}} x(T)=I^{\delta_{1}} h_{1}(T, x(T)), \quad \rho_{1} \in(1,2), m_{1}, n_{1} \in \mathbb{R} \\
x^{(k)}\left(t_{0}\right)=I^{\delta_{k}} h_{k}(T, x(T)), \quad k=2,3, \ldots, n-1, n>2,
\end{array}\right.
$$

where $m_{1}, n_{0} \neq 0, D^{\alpha}$ denotes the Caputo fractional derivative and $f, g, h_{k}:\left[t_{0}, T\right] \times \mathbb{R} \rightarrow \mathbb{R}$, $k=0,1, \ldots, n-1$, are given continuous functions, and $\delta_{0}, \ldots, \delta_{n-1}>0$. Note that the novelty of the paper in the above system is that we shall investigate the qualitative criteria for two positive solutions to a new hybrid system with the finite number of integro-differential boundary conditions in terminal points with the help of a complicated case of fixed point techniques due to Avery-Anderson-Henderson. By taking different values for existing parameters and functions, one can get some well-known FDEs studied in the previous research works.

The other five sections of the manuscript are summarized as follows: In Sect. 2, we offer basic preliminaries of results in fractional calculus and fixed point theories. In Sect. 3, the solution of the fractional linear model of (1) is obtained. Therefore, an application of Banach fixed point theorem on the integral solution for system (1) implies the existence of one and only one solution of the system. In Sect. 4, to apply Avery-Anderson-Henderson 
fixed point theorem, we obtain sufficient criteria and conditions for the positivity and existence of at least two of them for fractional hybrid system (1) by showing the complete continuity of the operator that represents the integral solution of the system. In Sect. 5, the UH stability of the solution is investigated and sufficient conditions for this kind of stability are obtained. Finally, in Sect. 6, we design an example to ensure the consistency of the results. The conclusion section closes this paper.

\section{Preliminaries and notations}

We again introduce several specifications and facts about fractional calculus and topics of fixed point theorems.

Definition 2.1 ([49]) The Riemann-Liouville (left-sided) fractional integral of a realvalued function $\phi \in C\left[t_{0}, T\right]$ is introduced as

$$
I^{q} \phi(t)=\frac{1}{\Gamma(q)} \int_{t_{0}}^{t}(t-s)^{q-1} \phi(s) d s, \quad q>0
$$

if it exists.

Definition 2.2 ([49]) The Caputo fractional derivative of order $q \in(n-1, n]$ for $\phi \in$ $C^{n}\left[t_{0}, T\right]$ is defined as

$$
D^{q} \phi(t)= \begin{cases}I^{n-q} \phi^{(n)}(t), & n-1<q<n, \\ \phi^{(n)}(t), & q=n,\end{cases}
$$

if it exists.

Lemma 2.3 ([49]) Let $n-1<q<n$, then

$$
I^{q} D^{q} \phi(t)=\phi(t)+a_{0}+a_{1}\left(t-t_{0}\right)+a_{2}\left(t-t_{0}\right)^{2}+\cdots+a_{n-1}\left(t-t_{0}\right)^{n-1}
$$

for $a_{k} \in \mathbb{R}, k=0,1, \ldots, n-1$.

For example, the $\gamma$ th Caputo derivative of $\phi(t)=\left(t-t_{0}\right)^{\zeta}$ is given by

$$
D^{\gamma}\left(t-t_{0}\right)^{\zeta}= \begin{cases}\frac{\Gamma(\zeta+1)}{\Gamma(\zeta-\gamma+1)}\left(t-t_{0}\right)^{\zeta-\gamma}, & n-1<\gamma<n, n-1<\zeta, \\ 0, & \zeta \leq n-1 .\end{cases}
$$

Theorem 2.4 (Banach principle [24]) Let $(E,\|\cdot\|)$ be a Banach space and $\Omega$ be a closed and bounded subset of $E$. If $\Psi: \Omega \rightarrow \Omega$ is a contraction operator, then $\Psi$ has a unique fixed point in $\Omega$. We mean by a contraction that it is an operator $\Psi$ that satisfies

$$
\|\Psi x-\Psi y\| \leq k\|x-y\|, \quad k \in(0,1), x, y \in \Omega .
$$

Theorem 2.5 (Avery-Anderson-Henderson theorem [43]) Consider $(E,\|\cdot\|)$ as a Banach space, $P \in E$ as a cone, and $\mu$ and $\phi$ as two increasing nonnegative continuous functionals on $P$, and let $\omega$ be a nonnegative continuous functional on $P$ with $\omega(0)=0$ provided that, 
for some $r_{3}>0$ and $M>0$, the inequalities $\phi(x) \leq \omega(x) \leq \mu(x)$ and $\|x\| \leq M \phi(x)$ fulfill $\forall x \in \overline{P\left(\phi, r_{3}\right)}$, in which $P\left(\phi, r_{3}\right)=\left\{x \in P: \phi(x)<r_{3}\right\}$. Let positive numbers $r_{1}<r_{2}<r_{3}$ exist so that $\omega(l x) \leq l \omega(x)$ for $0 \leq l \leq 1$, and $x \in \partial P\left(\omega, r_{2}\right)$. If $\Psi: \overline{P\left(\phi, r_{3}\right)} \rightarrow P$ is an operator with the complete continuity property satisfying:

(C1) $\phi(\Psi x)>r_{3}, \forall x \in \partial P\left(\phi, r_{3}\right)$;

(C2) $\omega(\Psi x)<r_{2}, \forall x \in \partial P\left(\omega, r_{2}\right)$;

(C3) $P\left(\mu, r_{1}\right) \neq \emptyset$, and $\mu(\Psi x)>r_{1}, \forall x \in \partial P\left(\mu, r_{1}\right)$,

then $\Psi$ admits at least two fixed points $x_{1}$ and $x_{2}$ provided that $r_{1}<\mu\left(x_{1}\right)$ with $\omega\left(x_{1}\right)<r_{2}$ and $r_{2}<\omega\left(x_{2}\right)$ with $\phi\left(x_{2}\right)<r_{3}$.

\section{Results regarding unique solution}

We obtain firstly a solution of the corresponding linear system of (1).

Theorem 3.1 Let $m_{1}, n_{0} \neq 0$ and $\delta_{0}, \ldots, \delta_{n-1}>0$. Then the linear hybrid fractional boundary value problem $(F B V P)$

$$
\left\{\begin{array}{l}
D^{\alpha}(x(t)-g(t))=f(t), \quad t \in\left(t_{0}, T\right), \alpha>2, \\
m_{0} x\left(t_{0}\right)+n_{0} D^{\rho_{0}} x(T)=I^{\delta_{0}} h_{0}(T), \quad \rho_{0} \in(0,1), m_{0}, n_{0} \in \mathbb{R}, \\
m_{1} x\left(t_{0}\right)+n_{1} D^{\rho_{1}} x(T)=I^{\delta_{1}} h_{1}(T), \quad \rho_{1} \in(1,2), m_{1}, n_{1} \in \mathbb{R}, \\
x^{(k)}\left(t_{0}\right)=I^{\delta_{k}} h_{k}(T), \quad k=2,3, \ldots, n-1, n \geq 3,
\end{array}\right.
$$

has an integral solution in the following form:

$$
\begin{aligned}
x(t)= & I^{\alpha} f(t)+g(t)-g\left(t_{0}\right) \\
& +\frac{\left(t-t_{0}\right) \Gamma\left(2-\rho_{0}\right)}{n_{0}\left(T-t_{0}\right)^{1-\rho_{0}}}\left(I^{\delta_{0}} h_{0}(T)-\frac{m_{0}}{m_{1}} I^{\delta_{1}} h_{1}(T)\right)+\frac{1}{m_{1}} I^{\delta_{1}} h_{1}(T) \\
& +\frac{\left(t-t_{0}\right) \Gamma\left(2-\rho_{0}\right)}{\left(T-t_{0}\right)^{1-\rho_{0}}}\left(\frac{n_{1} m_{0}}{m_{1} n_{0}} I^{\alpha-\rho_{1}} f(T)-I^{\alpha-\rho_{0}} f(T)\right)-\frac{n_{1}}{m_{1}} I^{\alpha-\rho_{1}} f(T) \\
& +\frac{\left(t-t_{0}\right) \Gamma\left(2-\rho_{0}\right)}{\left(T-t_{0}\right)^{1-\rho_{0}}}\left(\frac{n_{1} m_{0}}{m_{1} n_{0}} D^{\rho_{1}} g(T)-D^{\rho_{0}} g(T)\right)-\frac{n_{1}}{m_{1}} D^{\rho_{1}} g(T) \\
& +\sum_{k=2}^{n-1}\left[\frac { n _ { 1 } ( T - t _ { 0 } ) ^ { k - \rho _ { 1 } } } { m _ { 1 } \Gamma ( k - \rho _ { 1 } + 1 ) } \left[1-\frac{m_{0}}{n_{0}} \frac{\left(t-t_{0}\right) \Gamma\left(2-\rho_{0}\right)}{\left.\left(T-t_{0}\right)^{1-\rho_{0}}\right]-\frac{\left(t-t_{0}\right)^{k}}{k !}}\right.\right. \\
& \left.+\frac{\left(T-t_{0}\right)^{k-1}\left(t-t_{0}\right) \Gamma\left(2-\rho_{0}\right)}{\Gamma\left(k-\rho_{0}+1\right)}\right]\left[g^{(k)}\left(t_{0}\right)-I^{\delta_{k}} h_{k}(T)\right] .
\end{aligned}
$$

Proof Taking the fractional integral to both sides of differential equation (3) and using Lemma 2.3, we get

$$
x(t)=I^{\alpha} f(t)+g(t)+\sum_{k=0}^{n-1} c_{k}\left(t-t_{0}\right)^{k} .
$$


By definition of the Caputo derivative of the fractional order $\rho_{i}<\alpha$, and using equation (2), it becomes

$$
\begin{aligned}
D^{\rho_{i}} x(t) & =I^{\alpha-\rho_{i}} f(t)+D^{\rho_{i}} g(t)+\sum_{k=0}^{n-1} c_{k} D^{\rho_{i}}\left(t-t_{0}\right)^{k} \\
& =I^{\alpha-\rho_{i}} f(t)+D^{\rho_{i}} g(t)+\sum_{k=\left\lceil\rho_{i}\right\rceil}^{n-1} \frac{c_{k} \Gamma(k+1)}{\Gamma\left(k-\rho_{i}+1\right)}\left(t-t_{0}\right)^{k-\rho_{i}} .
\end{aligned}
$$

Particularly, we find

$$
D^{\rho_{0}} x(t)=I^{\alpha-\rho_{0}} f(t)+D^{\rho_{0}} g(t)+\sum_{k=1}^{n-1} \frac{c_{k} \Gamma(k+1)}{\Gamma\left(k-\rho_{0}+1\right)}\left(t-t_{0}\right)^{k-\rho_{0}}
$$

and

$$
D^{\rho_{1}} x(t)=I^{\alpha-\rho_{1}} f(t)+D^{\rho_{1}} g(t)+\sum_{k=2}^{n-1} \frac{c_{k} \Gamma(k+1)}{\Gamma\left(k-\rho_{1}+1\right)}\left(t-t_{0}\right)^{k-\rho_{1}} .
$$

Then

$$
x\left(t_{0}\right)=g\left(t_{0}\right)+c_{0}
$$

and

$$
D^{\rho_{i}} x\left(t_{0}\right)=D^{\rho_{i}} g\left(t_{0}\right) \quad(i \in\{0,1\}) .
$$

Also, we have

$$
x(T)=I^{\alpha} f(T)+g(T)+\sum_{k=0}^{n-1} c_{k}\left(T-t_{0}\right)^{k}
$$

and

$$
D^{\rho_{i}} x(T)=I^{\alpha-\rho_{i}} f(T)+D^{\rho_{i}} g(T)+\sum_{k=\left\lceil\rho_{i}\right\rceil}^{n-1} \frac{c_{k} \Gamma(k+1)}{\Gamma\left(k-\rho_{i}+1\right)}\left(T-t_{0}\right)^{k-\rho_{i}} .
$$

The boundary condition $m_{0} x\left(t_{0}\right)+n_{0} D^{\rho_{0}} x(T)=I^{\delta_{0}} h_{0}(T)$ gives

$$
\begin{aligned}
I^{\delta_{0}} h_{0}(T)= & m_{0} g\left(t_{0}\right)+m_{0} c_{0}+n_{0} I^{\alpha-\rho_{0}} f(T)+n_{0} D^{\rho_{0}} g(T) \\
& +n_{0} \sum_{k=1}^{n-1} \frac{c_{k} \Gamma(k+1)}{\Gamma\left(k-\rho_{0}+1\right)}\left(T-t_{0}\right)^{k-\rho_{0}},
\end{aligned}
$$

and the condition $m_{1} x\left(t_{0}\right)+n_{1} D^{\rho_{1}} x(T)=I^{\delta_{1}} h_{1}(T)$ implies

$$
\begin{aligned}
I^{\delta_{1}} h_{1}(T)= & m_{1} g\left(t_{0}\right)+m_{1} c_{0}+n_{1} I^{\alpha-\rho_{1}} f(T)+n_{1} D^{\rho_{1}} g(T) \\
& +n_{1} \sum_{k=2}^{n-1} \frac{c_{k} \Gamma(k+1)}{\Gamma\left(k-\rho_{1}+1\right)}\left(T-t_{0}\right)^{k-\rho_{1}} .
\end{aligned}
$$


The boundary condition $x^{(k)}\left(t_{0}\right)=I^{\delta_{k}} h_{k}(T), k=2,3, \ldots, n-1$, implies that

$$
c_{k}=\frac{1}{k !}\left[I^{\delta_{k}} h_{k}(T)-g^{(k)}\left(t_{0}\right)\right], \quad k=2,3, \ldots, n-1 .
$$

Substituting (8) into (6) and (7), we obtain

$$
\begin{aligned}
m_{0} c_{0} & +\frac{c_{1} n_{0}}{\Gamma\left(2-\rho_{0}\right)}\left(T-t_{0}\right)^{1-\rho_{0}} \\
= & I^{\delta_{0}} h_{0}(T)-m_{0} g\left(t_{0}\right)-n_{0} I^{\alpha-\rho_{0}} f(T)-n_{0} D^{\rho_{0}} g(T) \\
& +n_{0} \sum_{k=2}^{n-1} \frac{\left(T-t_{0}\right)^{k-\rho_{0}}}{\Gamma\left(k-\rho_{0}+1\right)}\left[g^{(k)}\left(t_{0}\right)-I^{\delta_{k}} h_{k}(T)\right]
\end{aligned}
$$

and

$$
\begin{aligned}
c_{0}= & m_{1}^{-1} I^{\delta_{1}} h_{1}(T)-g\left(t_{0}\right)-m_{1}^{-1} n_{1} I^{\alpha-\rho_{1}} f(T)-m_{1}^{-1} n_{1} D^{\rho_{1}} g(T) \\
& +m_{1}^{-1} n_{1} \sum_{k=2}^{n-1} \frac{\left(T-t_{0}\right)^{k-\rho_{1}}}{\Gamma\left(k-\rho_{1}+1\right)}\left[g^{(k)}\left(t_{0}\right)-I^{\delta_{k}} h_{k}(T)\right] .
\end{aligned}
$$

Then

$$
\begin{aligned}
c_{1}= & \frac{\Gamma\left(2-\rho_{0}\right)}{n_{0}\left(T-t_{0}\right)^{1-\rho_{0}}}\left(I^{\delta_{0}} h_{0}(T)-\frac{m_{0}}{m_{1}} I^{\delta_{1}} h_{1}(T)\right) \\
& -\frac{\Gamma\left(2-\rho_{0}\right)}{\left(T-t_{0}\right)^{1-\rho_{0}}}\left(I^{\alpha-\rho_{0}} f(T)-\frac{m_{0} n_{1}}{n_{0} m_{1}} I^{\alpha-\rho_{1}} f(T)\right) \\
& -\frac{\Gamma\left(2-\rho_{0}\right)}{\left(T-t_{0}\right)^{1-\rho_{0}}}\left(D^{\rho_{0}} g(T)-\frac{m_{0} n_{1}}{n_{0} m_{1}} D^{\rho_{1}} g(T)\right) \\
& +\frac{\Gamma\left(2-\rho_{0}\right)}{\left(T-t_{0}\right)^{1-\rho_{0}}} \sum_{k=2}^{n-1}\left(\frac{\left(T-t_{0}\right)^{k-\rho_{0}}}{\Gamma\left(k-\rho_{0}+1\right)}-\frac{m_{0} n_{1}\left(T-t_{0}\right)^{k-\rho_{1}}}{n_{0} m_{1} \Gamma\left(k-\rho_{1}+1\right)}\right) \\
& \times\left(g^{(k)}\left(t_{0}\right)-I^{\delta_{k}} h_{k}(T)\right) .
\end{aligned}
$$

Substituting the constants $c_{k}, k=0,1,2, \ldots, n-1$, into (5), we obtain solution (4), and the proof is finished.

If $\rho_{0}, \rho_{1} \in(0,1)$, then the integral solution (4) will be different. This case is explained in the next result.

Theorem 3.2 Let $\frac{m_{0} n_{1}}{n_{0} m_{1}} \neq \frac{\Gamma\left(2-\rho_{1}\right)}{\Gamma\left(2-\rho_{0}\right)}\left(T-t_{0}\right)^{\rho_{1}-\rho_{0}}$ and $\delta_{0}, \ldots, \delta_{n-1}>0$. Then the linear hybrid FBVP

$$
\left\{\begin{array}{l}
D^{\alpha}(x(t)-g(t))=f(t), \quad t \in\left(t_{0}, T\right), \alpha>2, \\
m_{0} x\left(t_{0}\right)+n_{0} D^{\rho_{0}} x(T)=I^{\delta_{0}} h_{0}(T), \quad \rho_{0} \in(0,1), m_{0}, n_{0} \in \mathbb{R}, \\
m_{1} x\left(t_{0}\right)+n_{1} D^{\rho_{1}} x(T)=I^{\delta_{1}} h_{1}(T), \quad \rho_{1} \in(0,1), m_{1}, n_{1} \in \mathbb{R}, \\
x^{(k)}\left(t_{0}\right)=I^{\delta_{k}} h_{k}(T), \quad k=2,3, \ldots, n-1, n \geq 3,
\end{array}\right.
$$


has an integral solution in the following form:

$$
\begin{aligned}
& x(t)=I^{\alpha} f(t)+g(t)-g\left(t_{0}\right) \\
& +\frac{\Gamma\left(2-\rho_{1}\right)\left(m_{0}\left(t-t_{0}\right) \Gamma\left(2-\rho_{0}\right)-n_{0}\left(T-t_{0}\right)^{1-\rho_{0}}\right)}{n_{1} m_{0} \Gamma\left(2-\rho_{0}\right)\left(T-t_{0}\right)^{1-\rho_{1}}-n_{0} m_{1} \Gamma\left(2-\rho_{1}\right)\left(T-t_{0}\right)^{1-\rho_{0}}} I^{\delta_{1}} h_{1}(T) \\
& +\frac{\Gamma\left(2-\rho_{0}\right)\left(n_{1}\left(T-t_{0}\right)^{1-\rho_{1}}-m_{1}\left(t-t_{0}\right) \Gamma\left(2-\rho_{1}\right)\right)}{n_{1} m_{0} \Gamma\left(2-\rho_{0}\right)\left(T-t_{0}\right)^{1-\rho_{1}}-n_{0} m_{1} \Gamma\left(2-\rho_{1}\right)\left(T-t_{0}\right)^{1-\rho_{0}}} I^{\delta_{0}} h_{0}(T) \\
& +\frac{n_{0} \Gamma\left(2-\rho_{0}\right)\left(m_{1}\left(t-t_{0}\right) \Gamma\left(2-\rho_{1}\right)-n_{1}\left(T-t_{0}\right)^{1-\rho_{1}}\right)}{n_{1} m_{0} \Gamma\left(2-\rho_{0}\right)\left(T-t_{0}\right)^{1-\rho_{1}}-n_{0} m_{1} \Gamma\left(2-\rho_{1}\right)\left(T-t_{0}\right)^{1-\rho_{0}}} I^{\alpha-\rho_{0}} f(T) \\
& +\frac{n_{1} \Gamma\left(2-\rho_{1}\right)\left(n_{0}\left(T-t_{0}\right)^{1-\rho_{0}}-m_{0}\left(t-t_{0}\right) \Gamma\left(2-\rho_{0}\right)\right)}{n_{1} m_{0} \Gamma\left(2-\rho_{0}\right)\left(T-t_{0}\right)^{1-\rho_{1}}-n_{0} m_{1} \Gamma\left(2-\rho_{1}\right)\left(T-t_{0}\right)^{1-\rho_{0}}} I^{\alpha-\rho_{1}} f(T) \\
& +\frac{n_{0} \Gamma\left(2-\rho_{0}\right)\left(m_{1}\left(t-t_{0}\right) \Gamma\left(2-\rho_{1}\right)-n_{1}\left(T-t_{0}\right)^{1-\rho_{1}}\right)}{n_{1} m_{0} \Gamma\left(2-\rho_{0}\right)\left(T-t_{0}\right)^{1-\rho_{1}}-n_{0} m_{1} \Gamma\left(2-\rho_{1}\right)\left(T-t_{0}\right)^{1-\rho_{0}}} D^{\rho_{0}} g(T) \\
& +\frac{n_{1} \Gamma\left(2-\rho_{1}\right)\left(n_{0}\left(T-t_{0}\right)^{1-\rho_{0}}-m_{0}\left(t-t_{0}\right) \Gamma\left(2-\rho_{0}\right)\right)}{n_{1} m_{0} \Gamma\left(2-\rho_{0}\right)\left(T-t_{0}\right)^{1-\rho_{1}}-n_{0} m_{1} \Gamma\left(2-\rho_{1}\right)\left(T-t_{0}\right)^{1-\rho_{0}}} D^{\rho_{1}} g(T) \\
& +\sum_{k=2}^{n-1}\left[\frac{\left(t-t_{0}\right) \Gamma\left(2-\rho_{0}\right) \Gamma\left(2-\rho_{1}\right)\left(\frac{n_{0} m_{1}\left(T-t_{0}\right)^{k-\rho_{0}}}{\Gamma\left(k-\rho_{0}+1\right)}-\frac{m_{0} n_{1}\left(T-t_{0}\right)^{k-\rho_{1}}}{\Gamma\left(k-\rho_{1}+1\right)}\right)}{n_{1} m_{0} \Gamma\left(2-\rho_{0}\right)\left(T-t_{0}\right)^{1-\rho_{1}}-n_{0} m_{1} \Gamma\left(2-\rho_{1}\right)\left(T-t_{0}\right)^{1-\rho_{0}}}+\frac{\left(t-t_{0}\right)^{k}}{k !}\right.
\end{aligned}
$$

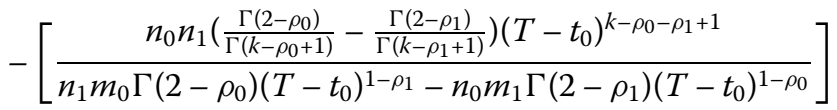

$$
\begin{aligned}
& \times\left[I^{\delta_{k}} h_{k}(T)-g^{(k)}\left(t_{0}\right)\right] \text {. }
\end{aligned}
$$

Proof We sketch the proof. Equation (7) will become

$$
\begin{aligned}
I^{\delta_{1}} h_{1}(T)= & m_{1} g\left(t_{0}\right)+m_{1} c_{0}+n_{1} I^{\alpha-\rho_{1}} f(T)+n_{1} D^{\rho_{1}} g(T) \\
& +n_{1} \sum_{k=1}^{n-1} \frac{c_{k} \Gamma(k+1)}{\Gamma\left(k-\rho_{1}+1\right)}\left(T-t_{0}\right)^{k-\rho_{1}} .
\end{aligned}
$$

Solving equations (6) and (11) and taking into account (8), we deduce that

$$
\begin{aligned}
c_{1}= & \frac{\Gamma\left(2-\rho_{0}\right) \Gamma\left(2-\rho_{1}\right)\left(m_{0} I^{\delta_{1}} h_{1}(T)-m_{1} I^{\delta_{0}} h_{0}(T)\right)}{n_{1} m_{0} \Gamma\left(2-\rho_{0}\right)\left(T-t_{0}\right)^{1-\rho_{1}}-n_{0} m_{1} \Gamma\left(2-\rho_{1}\right)\left(T-t_{0}\right)^{1-\rho_{0}}} \\
& +\frac{\Gamma\left(2-\rho_{0}\right) \Gamma\left(2-\rho_{1}\right)\left(n_{0} m_{1} I^{\alpha-\rho_{0}} f(T)-m_{0} n_{1} I^{\alpha-\rho_{1}} f(T)\right)}{n_{1} m_{0} \Gamma\left(2-\rho_{0}\right)\left(T-t_{0}\right)^{1-\rho_{1}}-n_{0} m_{1} \Gamma\left(2-\rho_{1}\right)\left(T-t_{0}\right)^{1-\rho_{0}}} \\
& +\frac{\Gamma\left(2-\rho_{0}\right) \Gamma\left(2-\rho_{1}\right)\left(n_{0} m_{1} D^{\rho_{0}} g(T)-m_{0} n_{1} D^{\rho_{1}} g(T)\right)}{n_{1} m_{0} \Gamma\left(2-\rho_{0}\right)\left(T-t_{0}\right)^{1-\rho_{1}}-n_{0} m_{1} \Gamma\left(2-\rho_{1}\right)\left(T-t_{0}\right)^{1-\rho_{0}}} \\
& +\frac{\Gamma\left(2-\rho_{0}\right) \Gamma\left(2-\rho_{1}\right)}{n_{1} m_{0} \Gamma\left(2-\rho_{0}\right)\left(T-t_{0}\right)^{1-\rho_{1}}-n_{0} m_{1} \Gamma\left(2-\rho_{1}\right)\left(T-t_{0}\right)^{1-\rho_{0}}} \\
& \times \sum_{k=2}^{n-1}\left(\frac{n_{0} m_{1}\left(T-t_{0}\right)^{k-\rho_{0}}}{\Gamma\left(k-\rho_{0}+1\right)}-\frac{m_{0} n_{1}\left(T-t_{0}\right)^{k-\rho_{1}}}{\Gamma\left(k-\rho_{1}+1\right)}\right)\left[I^{\delta_{k}} h_{k}(T)-g^{(k)}\left(t_{0}\right)\right]
\end{aligned}
$$


and

$$
\begin{aligned}
c_{0}= & -g\left(t_{0}\right)+\frac{n_{1} \Gamma\left(2-\rho_{0}\right)\left(T-t_{0}\right)^{1-\rho_{1}} I^{\delta_{0}} h_{0}(T)-n_{0} \Gamma\left(2-\rho_{1}\right)\left(T-t_{0}\right)^{1-\rho_{0}} I^{\delta_{1}} h_{1}(T)}{n_{1} m_{0} \Gamma\left(2-\rho_{0}\right)\left(T-t_{0}\right)^{1-\rho_{1}}-n_{0} m_{1} \Gamma\left(2-\rho_{1}\right)\left(T-t_{0}\right)^{1-\rho_{0}}} \\
& -n_{0} n_{1} \frac{\Gamma\left(2-\rho_{0}\right)\left(T-t_{0}\right)^{1-\rho_{1}} I^{\alpha-\rho_{0}} f(T)-\Gamma\left(2-\rho_{1}\right)\left(T-t_{0}\right)^{1-\rho_{0}} I^{\alpha-\rho_{1}} f(T)}{m_{0} n_{1} \Gamma\left(2-\rho_{0}\right)\left(T-t_{0}\right)^{1-\rho_{1}}-n_{0} m_{1} \Gamma\left(2-\rho_{1}\right)\left(T-t_{0}\right)^{1-\rho_{0}}} \\
& -n_{0} n_{1} \frac{\Gamma\left(2-\rho_{0}\right)\left(T-t_{0}\right)^{1-\rho_{1}} D^{\rho_{0}} g(T)-\Gamma\left(2-\rho_{1}\right)\left(T-t_{0}\right)^{1-\rho_{0}} D^{\rho_{1}} g(T)}{m_{0} n_{1} \Gamma\left(2-\rho_{0}\right)\left(T-t_{0}\right)^{1-\rho_{1}}-n_{0} m_{1} \Gamma\left(2-\rho_{1}\right)\left(T-t_{0}\right)^{1-\rho_{0}}} \\
& -n_{0} n_{1} \sum_{k=2}^{n-1} \frac{\left(\frac{\Gamma\left(2-\rho_{0}\right)}{\Gamma\left(k-\rho_{0}+1\right)}-\frac{\Gamma\left(2-\rho_{1}\right)}{\Gamma\left(k-\rho_{1}+1\right)}\right)\left(T-t_{0}\right)^{k-\rho_{0}-\rho_{1}+1}\left[I^{\delta_{k}} h_{k}(T)-g^{(k)}\left(t_{0}\right)\right]}{n_{1} m_{0} \Gamma\left(2-\rho_{0}\right)\left(T-t_{0}\right)^{1-\rho_{1}}-n_{0} m_{1} \Gamma\left(2-\rho_{1}\right)\left(T-t_{0}\right)^{1-\rho_{0}}} .
\end{aligned}
$$

Substituting the constants $c_{k}, k=0,1,2, \ldots, n-1$, into (5), we obtain solution (10), and this completes our proof.

Now, by Theorem 2.4, we prove the existence of a unique solution to system (1). For this, define an operator $\Psi: C\left(\left[t_{0}, T\right], \mathbb{R}\right) \rightarrow \mathbb{R}$ provided

$$
\begin{aligned}
\Psi x(t)= & I^{\alpha} f(t, x(t))+g(t, x(t))-g\left(t_{0}, x\left(t_{0}\right)\right) \\
& +\frac{\left(t-t_{0}\right) \Gamma\left(2-\rho_{0}\right)}{n_{0}\left(T-t_{0}\right)^{1-\rho_{0}}}\left(I^{\delta_{0}} h_{0}(T, x(T))-\frac{m_{0}}{m_{1}} I^{\delta_{1}} h_{1}(T, x(T))\right)+\frac{1}{m_{1}} I^{\delta_{1}} h_{1}(T, x(T)) \\
& +\frac{\left(t-t_{0}\right) \Gamma\left(2-\rho_{0}\right)}{\left(T-t_{0}\right)^{1-\rho_{0}}}\left(\frac{n_{1} m_{0}}{m_{1} n_{0}} I^{\alpha-\rho_{1}} f(T, x(T))-I^{\alpha-\rho_{0}} f(T, x(T))\right) \\
& -\frac{n_{1}}{m_{1}} I^{\alpha-\rho_{1}} f(T, x(T)) \\
& +\frac{\left(t-t_{0}\right) \Gamma\left(2-\rho_{0}\right)}{\left(T-t_{0}\right)^{1-\rho_{0}}\left(\frac{n_{1} m_{0}}{m_{1} n_{0}} D^{\rho_{1}} g(T, x(T))-D^{\rho_{0}} g(T, x(T))\right)} \\
& -\frac{n_{1}}{m_{1}} D^{\rho_{1}} g(T, x(T)) \\
& +\sum_{k=2}^{n-1}\left[\frac{n_{1}\left(T-t_{0}\right)^{k-\rho_{1}}}{m_{1} \Gamma\left(k-\rho_{1}+1\right)}\left[1-\frac{m_{0}}{n_{0}} \frac{\left(t-t_{0}\right) \Gamma\left(2-\rho_{0}\right)}{\left(T-t_{0}\right)^{1-\rho_{0}}}\right]-\frac{\left(t-t_{0}\right)^{k}}{k !}\right. \\
& \left.+\frac{\left(T-t_{0}\right)^{k-1}\left(t-t_{0}\right) \Gamma\left(2-\rho_{0}\right)}{\Gamma\left(k-\rho_{0}+1\right)}\right]\left[g^{(k)}\left(t_{0}, x\left(t_{0}\right)\right)-I^{\delta_{k}} h_{k}(T, x(T))\right], \quad m_{1}, n_{0} \neq 0 .
\end{aligned}
$$

The required criterion for finding a unique solution of the nonlinear hybrid FBVP (1) is given in the next result.

\section{Theorem 3.3 Assume}

$[\mathrm{H} 1] f, g^{(k)}, h_{k}:\left[t_{0}, T\right] \times C\left(\left[t_{0}, T\right], \mathbb{R}\right) \rightarrow \mathbb{R}, k=0,1, \ldots, n-1$, are continuous functions such that

$$
\left\{\begin{array}{l}
\left|f\left(t, \mathrm{u}_{1}\right)-f\left(s, \mathrm{v}_{1}\right)\right| \leq C_{f}\left|\mathrm{u}_{1}-\mathrm{v}_{1}\right| \\
\left|g^{(k)}\left(t, \mathrm{u}_{1}\right)-g^{(k)}\left(s, \mathrm{v}_{1}\right)\right| \leq C_{g^{(k)}}\left|\mathrm{u}_{1}-\mathrm{v}_{1}\right| \\
\left|h_{k}\left(t, \mathrm{u}_{1}\right)-h_{k}\left(s, \mathrm{v}_{1}\right)\right| \leq C_{h_{k}}\left|\mathrm{u}_{1}-\mathrm{v}_{1}\right|
\end{array}\right.
$$

where $t, s \in\left[t_{0}, T\right], \mathrm{u}_{1}, \mathrm{v}_{1} \in C\left(\left[t_{0}, T\right], \mathbb{R}\right), C_{f}, C_{g^{(k)}}, C_{h_{k}}$ are nonnegative constants. 
Then the hybrid FBVP system (1) admits one and only one solution provided that $\Delta<1$, where

$$
\begin{aligned}
\Delta= & \frac{C_{f}\left(T-t_{0}\right)^{\alpha}}{\Gamma(\alpha+1)}+2 C_{g}+\frac{\left(T-t_{0}\right)^{\rho_{0}} \Gamma\left(2-\rho_{0}\right)}{\left|n_{0}\right|} \\
& \times\left[\frac{\left(T-t_{0}\right)^{\delta_{0}} C_{h_{0}}}{\Gamma\left(\delta_{0}+1\right)}+\frac{\left|m_{0}\right|\left(T-t_{0}\right)^{\delta_{1}} C_{h_{1}}}{\left|m_{1}\right| \Gamma\left(\delta_{1}+1\right)}\right] \\
& +\frac{\left(T-t_{0}\right)^{\delta_{1}} C_{h_{1}}}{\left|m_{1}\right| \Gamma\left(\delta_{1}+1\right)}+\left(T-t_{0}\right)^{\rho_{0}} \Gamma\left(2-\rho_{0}\right) C_{f} \\
& \times\left[\frac{\left|n_{1} m_{0}\right|\left(T-t_{0}\right)^{\alpha-\rho_{1}}}{\left|m_{1} n_{0}\right| \Gamma\left(\alpha-\rho_{1}+1\right)}+\frac{\left(T-t_{0}\right)^{\alpha-\rho_{0}}}{\Gamma\left(\alpha-\rho_{0}+1\right)}\right]+\frac{\left|n_{1}\right|\left(T-t_{0}\right)^{\alpha-\rho_{1}} C_{f}}{\left|m_{1}\right| \Gamma\left(\alpha-\rho_{1}+1\right)} \\
& +\left(T-t_{0}\right)^{\rho_{1}} \Gamma\left(2-\rho_{0}\right)\left[\frac{\left|n_{1} m_{0}\right| C_{g^{(2)}}\left(T-t_{0}\right)^{2-\rho_{1}}}{\left|m_{1} n_{0}\right| \Gamma\left(3-\rho_{1}\right)}+\frac{\left(T-t_{0}\right)^{1-\rho_{0}} C_{g^{(1)}}}{\Gamma\left(2-\rho_{0}\right)}\right] \\
& +\frac{\left|n_{1}\right| C_{g^{(2)}}\left(T-t_{0}\right)^{2-\rho_{1}}}{\left|m_{1}\right| \Gamma\left(3-\rho_{1}\right)} \\
& +\sum_{k=2}^{n-1} \max _{t \in\left[t_{0}, T\right]} \mid \frac{n_{1}\left(T-t_{0}\right)^{k-\rho_{1}}}{m_{1} \Gamma\left(k-\rho_{1}+1\right)}\left[1-\frac{m_{0}}{n_{0}} \frac{\left(t-t_{0}\right) \Gamma\left(2-\rho_{0}\right)}{\left.\left(T-t_{0}\right)^{1-\rho_{0}}\right]}\right. \\
& -\frac{\left(t-t_{0}\right)^{k}}{k !}+\frac{\left(T-t_{0}\right)^{k-1}\left(t-t_{0}\right) \Gamma\left(2-\rho_{0}\right)}{\Gamma\left(k-\rho_{0}+1\right)} \mid\left[C_{g^{(k)}}+\frac{\left(T-t_{0}\right)^{\delta_{k}} C_{h_{k}}}{\Gamma\left(\delta_{k}+1\right)}\right] .
\end{aligned}
$$

Proof Let $\Omega$ be any closed bounded subset of $E$. Then the continuity of $\Psi$ is followed by that of constitutive functions and Lebesgue dominated convergence theorem. By enlarging the set $\Omega$, one can deduce that $\Psi$ maps $\Omega$ into itself. We need to show the contraction property of the operator $\Psi$. For this, let $x, y \in \Omega$, then

$$
\begin{aligned}
\mid \Psi y(t) & -\Psi x(t) \mid \\
\leq & \frac{1}{\Gamma(\alpha)} \int_{t_{0}}^{t}(t-s)^{\alpha-1}|f(s, y(s))-f(s, x(s))| d s \\
& +|g(t, y(t))-g(t, x(t))|+\left|g\left(t_{0}, y\left(t_{0}\right)\right)-g\left(t_{0}, x\left(t_{0}\right)\right)\right| \\
& +\frac{\left(t-t_{0}\right) \Gamma\left(2-\rho_{0}\right)}{\left|n_{0}\right|\left(T-t_{0}\right)^{1-\rho_{0}}}\left[\frac{1}{\Gamma\left(\delta_{0}\right)} \int_{t_{0}}^{T}(T-s)^{\delta_{0}-1}\left|h_{0}(s, y(s))-h_{0}(s, x(s))\right| d s\right. \\
& \left.+\frac{\left|m_{0}\right|}{\left|m_{1}\right| \Gamma\left(\delta_{1}\right)} \int_{t_{0}}^{T}(T-s)^{\delta_{1}-1}\left|h_{1}(s, y(s))-h_{1}(s, x(s))\right| d s\right] \\
& +\frac{1}{\left|m_{1}\right| \Gamma\left(\delta_{1}\right)} \int_{t_{0}}^{T}(T-s)^{\delta_{1}-1}\left|h_{1}(s, y(s))-h_{1}(s, x(s))\right| d s \\
& +\frac{\left(t-t_{0}\right) \Gamma\left(2-\rho_{0}\right)}{\left(T-t_{0}\right)^{1-\rho_{0}}}\left[\frac{\left|n_{1} m_{0}\right|}{\left|m_{1} n_{0}\right| \Gamma\left(\alpha-\rho_{1}\right)} \int_{t_{0}}^{T}(T-s)^{\alpha-\rho_{1}-1}|f(s, y(s))-f(s, x(s))| d s\right. \\
& \left.+\frac{1}{\Gamma\left(\alpha-\rho_{0}\right)} \int_{t_{0}}^{T}(T-s)^{\alpha-\rho_{0}-1}|f(s, y(s))-f(s, x(s))| d s\right] \\
& +\frac{\left|n_{1}\right|}{\left|m_{1}\right| \Gamma\left(\alpha-\rho_{1}\right)} \int_{t_{0}}^{T}(T-s)^{\alpha-\rho_{1}-1}|f(s, y(s))-f(s, x(s))| d s
\end{aligned}
$$




$$
\begin{aligned}
& +\frac{\left(t-t_{0}\right) \Gamma\left(2-\rho_{0}\right)}{\left(T-t_{0}\right)^{1-\rho_{0}}}\left[\frac{\left|n_{1} m_{0}\right|}{\left|m_{1} n_{0}\right| \Gamma\left(2-\rho_{1}\right)} \int_{t_{0}}^{T}(T-s)^{1-\rho_{1}}\left|g^{\prime \prime}(s, y(s))-g^{\prime \prime}(s, x(s))\right| d s\right. \\
& \left.+\frac{1}{\Gamma\left(1-\rho_{0}\right)} \int_{t_{0}}^{T}(T-s)^{-\rho_{0}}\left|g^{\prime}(s, y(s))-g^{\prime}(s, x(s))\right| d s\right] \\
& +\frac{\left|n_{1}\right|}{\left|m_{1}\right| \Gamma\left(2-\rho_{1}\right)} \int_{t_{0}}^{T}(T-s)^{1-\rho_{1}}\left|g^{\prime \prime}(s, y(s))-g^{\prime \prime}(s, x(s))\right| d s \\
& +\sum_{k=2}^{n-1} \mid \frac{n_{1}\left(T-t_{0}\right)^{k-\rho_{1}}}{m_{1} \Gamma\left(k-\rho_{1}+1\right)}\left[1-\frac{m_{0}}{n_{0}} \frac{\left(t-t_{0}\right) \Gamma\left(2-\rho_{0}\right)}{\left(T-t_{0}\right)^{1-\rho_{0}}}\right]-\frac{\left(t-t_{0}\right)^{k}}{k !} \\
& +\frac{\left(T-t_{0}\right)^{k-1}\left(t-t_{0}\right) \Gamma\left(2-\rho_{0}\right)}{\Gamma\left(k-\rho_{0}+1\right)} \mid\left[\left|g^{(k)}\left(t_{0}, x\left(t_{0}\right)\right)-g^{(k)}\left(t_{0}, y\left(t_{0}\right)\right)\right|\right. \\
& \left.+\frac{1}{\Gamma\left(\delta_{k}\right)} \int_{t_{0}}^{T}(T-s)^{\delta_{k}-1}\left|h_{k}(s, y(s))-h_{k}(s, x(s))\right| d s\right] \\
& \leq \frac{C_{f}\left(T-t_{0}\right)^{\alpha}}{\Gamma(\alpha+1)}\|y-x\|+2 C_{g}\|y-x\| \\
& +\frac{\left(T-t_{0}\right)^{\rho_{0}} \Gamma\left(2-\rho_{0}\right)}{\left|n_{0}\right|}\left[\frac{\left(T-t_{0}\right)^{\delta_{0}} C_{h_{0}}}{\Gamma\left(\delta_{0}+1\right)}+\frac{\left|m_{0}\right|\left(T-t_{0}\right)^{\delta_{1}} C_{h_{1}}}{\left|m_{1}\right| \Gamma\left(\delta_{1}+1\right)}\right]\|y-x\| \\
& +\frac{\left(T-t_{0}\right)^{\delta_{1}} C_{h_{1}}}{\left|m_{1}\right| \Gamma\left(\delta_{1}+1\right)}\|y-x\| \\
& +\left(T-t_{0}\right)^{\rho_{0}} \Gamma\left(2-\rho_{0}\right) C_{f}\left[\frac{\left|n_{1} m_{0}\right|\left(T-t_{0}\right)^{\alpha-\rho_{1}}}{\left|m_{1} n_{0}\right| \Gamma\left(\alpha-\rho_{1}+1\right)}+\frac{\left(T-t_{0}\right)^{\alpha-\rho_{0}}}{\Gamma\left(\alpha-\rho_{0}+1\right)}\right]\|y-x\| \\
& +\frac{\left|n_{1}\right|\left(T-t_{0}\right)^{\alpha-\rho_{1}} C_{f}}{\left|m_{1}\right| \Gamma\left(\alpha-\rho_{1}+1\right)}\|y-x\| \\
& +\left(T-t_{0}\right)^{\rho_{1}} \Gamma\left(2-\rho_{0}\right)\left[\frac{\left|n_{1} m_{0}\right| C_{g^{(2)}}\left(T-t_{0}\right)^{2-\rho_{1}}}{\left|m_{1} n_{0}\right| \Gamma\left(3-\rho_{1}\right)}+\frac{\left(T-t_{0}\right)^{1-\rho_{0}} C_{g^{(1)}}}{\Gamma\left(2-\rho_{0}\right)}\right]\|y-x\| \\
& +\frac{\left|n_{1}\right| C_{g^{(2)}}\left(T-t_{0}\right)^{2-\rho_{1}}}{\left|m_{1}\right| \Gamma\left(3-\rho_{1}\right)}\|y-x\| \\
& +\sum_{k=2}^{n-1} \mid \frac{n_{1}\left(T-t_{0}\right)^{k-\rho_{1}}}{m_{1} \Gamma\left(k-\rho_{1}+1\right)}\left[1-\frac{m_{0}}{n_{0}} \frac{\left(t-t_{0}\right) \Gamma\left(2-\rho_{0}\right)}{\left(T-t_{0}\right)^{1-\rho_{0}}}\right]-\frac{\left(t-t_{0}\right)^{k}}{k !} \\
& +\frac{\left(T-t_{0}\right)^{k-1}\left(t-t_{0}\right) \Gamma\left(2-\rho_{0}\right)}{\Gamma\left(k-\rho_{0}+1\right)} \mid\left[C_{g^{(k)}}+\frac{\left(T-t_{0}\right)^{\delta_{k}} C_{h_{k}}}{\Gamma\left(\delta_{k}+1\right)}\right]\|y-x\|=\Delta\|y-x\|,
\end{aligned}
$$

where $\Delta$ is introduced in (12). Thus, the operator $\Psi$ satisfies the contraction property of Banach fixed point Theorem 2.4 with constant $\Delta<1$. Hence $\Psi$ admits a fixed point in $\Omega$, which is the same unique solution of hybrid FBVP (1).

\section{Results regarding two positive solutions}

We establish sufficient conditions of the existence of two positive solutions of system (1) with the help of the existing hypotheses on cones presented in Theorem 2.5 due to AveryAnderson-Henderson.

Define the cone $P=\left\{x \in C\left(\left[t_{0}, T\right], \mathbb{R}\right): x(t) \geq 0\right\}$. We want to obtain firstly sufficient conditions to make $\Psi x \in P$, whenever $x \in P$.

The next assumptions are essential for the coming results. 
[H2] Assume that

$$
\left\{\begin{array}{l}
m_{0}, n_{1} \leq 0, \quad m_{1}, n_{0}>0 \\
\frac{\Gamma\left(3-\rho_{1}\right)}{\Gamma\left(3-\rho_{0}\right)}<\frac{m_{0} n_{1}}{n_{0} m_{1}}\left(T-t_{0}\right)^{\rho_{0}-\rho_{1}} \\
f(t, x(t)) \geq 0 \\
g(t, x(t)) \geq g\left(t_{0}, x\left(t_{0}\right)\right) \geq 0, \quad t \in\left[t_{0}, T\right] \\
\frac{n_{1} m_{0}}{m_{1} n_{0}} I^{\alpha-\rho_{1}} f(T, x(T)) \geq I^{\alpha-\rho_{0}} f(T, x(T)) \\
K g(\kappa, x(\kappa)) \geq \frac{n_{1} m_{0}}{m_{1} n_{0}} D^{\rho_{1}} g(T, x(T)) \geq D^{\rho_{0}} g(T, x(T)) \geq 0, \quad K>0, \kappa \in\left[t_{0}, T\right] \\
0 \leq g^{(k)}\left(t_{0}, x\left(t_{0}\right)\right) \leq I^{\delta_{k}} h_{k}(T, x(T)), \quad k=2,3, \ldots, n-1 .
\end{array}\right.
$$

[H3] $f, g^{(k)}, h_{k}:\left[t_{0}, T\right] \times P \rightarrow \mathbb{R}, k=0,1, \ldots, n-1$, are all bounded and continuous functions. Moreover, for $r>0$, there exist a positive real number $L_{r}$ and a continuous nonnegative function $\mathfrak{g}$ so that $\forall(t, x),(s, y) \in\left[t_{0}, T\right] \times[0, r]$,

$$
|g(t, x)-g(s, y)| \leq L_{r}|\mathfrak{g}(x)-\mathfrak{g}(y)|
$$

Lemma 4.1 If $[H 2]$ holds and $x \in P$, then $\Psi x \in P$.

Proof For any $t \in\left[t_{0}, T\right]$, let

$$
\xi_{k}(t)=C_{1}\left(1-C_{2}\left(t-t_{0}\right)\right)-\frac{\left(t-t_{0}\right)^{k}}{k !}+C_{3}\left(t-t_{0}\right),
$$

where

$$
C_{1}=\frac{n_{1}\left(T-t_{0}\right)^{k-\rho_{1}}}{m_{1} \Gamma\left(k-\rho_{1}+1\right)}, \quad C_{2}=\frac{m_{0}}{n_{0}} \frac{\Gamma\left(2-\rho_{0}\right)}{\left(T-t_{0}\right)^{1-\rho_{0}}}
$$

and

$$
C_{3}=\frac{\left(T-t_{0}\right)^{k-1} \Gamma\left(2-\rho_{0}\right)}{\Gamma\left(k-\rho_{0}+1\right)} .
$$

We first need to show that $\xi_{k}(t) \leq 0$, that is,

$$
C_{1}+\left(C_{3}-C_{1} C_{2}\right)\left(t-t_{0}\right) \leq \frac{\left(t-t_{0}\right)^{k}}{k !}, \quad t \in\left[t_{0}, T\right] .
$$

Therefore, it suffices to show that $C_{1}+\left(C_{3}-C_{1} C_{2}\right)\left(t-t_{0}\right) \leq 0$. Notice that $\xi_{k}\left(t_{0}\right)=C_{1}<0$, then we need to show that $C_{3} \leq C_{1} C_{2}$, that is,

$$
\frac{\Gamma\left(k-\rho_{1}+1\right)}{\Gamma\left(k-\rho_{0}+1\right)}<\frac{m_{0} n_{1}}{n_{0} m_{1}}\left(T-t_{0}\right)^{\rho_{0}-\rho_{1}}, \quad k=2,3, \ldots, n-1 .
$$

By induction on $k$, it is obvious, by assumption, that it is true for $k=2$. We assume it is true for the case $k$ and show it for the case $k+1$. We have

$$
\begin{aligned}
\frac{\Gamma\left(k-\rho_{1}+2\right)}{\Gamma\left(k-\rho_{0}+2\right)} & =\frac{\left(k-\rho_{1}+1\right) \Gamma\left(k-\rho_{1}+1\right)}{\left(k-\rho_{0}+1\right) \Gamma\left(k-\rho_{0}+1\right)} \\
& <\frac{m_{0} n_{1}\left(k-\rho_{1}+1\right)}{n_{0} m_{1}\left(k-\rho_{0}+1\right)}\left(T-t_{0}\right)^{\rho_{0}-\rho_{1}}<\frac{m_{0} n_{1}}{n_{0} m_{1}}\left(T-t_{0}\right)^{\rho_{0}-\rho_{1}} .
\end{aligned}
$$


We deduce now

$$
\sum_{k=2}^{n-1} \xi_{k}(t)\left[g^{(k)}\left(t_{0}, x\left(t_{0}\right)\right)-I^{\delta_{k}} h_{k}(T, x(T))\right] \geq 0 .
$$

The remainder of the proof is obvious by the given assumptions. Hence, the result follows.

Lemma 4.2 If $[\mathrm{H} 2]$ and $[\mathrm{H} 3]$ are fulfilled, then $\Psi: P \rightarrow P$ admits the complete continuity property.

Proof Define a bounded subset $B_{r}=\{x \in P: x(t) \leq r\}$ of $P$, and let

$$
\left\{\begin{array}{l}
\max _{t \in\left[t_{0}, T\right], x \in[0, r]} f(t, x) \leq L_{f} \\
\max _{t \in\left[t_{0}, T\right], x \in[0, r]} g^{(k)}(t, x) \leq L_{g^{(k)}} \\
\max _{t \in\left[t_{0}, T\right], x \in[0, r]} h_{k}(t, x) \leq L_{h_{k}}
\end{array}\right.
$$

for positive constants $L_{f}, L_{g^{(k)}}$, and $L_{h_{k}}, k=0,1,2, \ldots, n-1$. The proof consists of three steps.

(Step 1) $\Psi$ is a continuous operator.

Assuming $x \in P$, then by Lemma 4.1, $\Psi x \in P$ which implies that $\Psi: P \rightarrow P$. Let $\left\{x_{m}\right\}$ be a sequence in the cone $P$ such that $\lim _{m \rightarrow \infty} x_{m}=x$ in $P$. The continuity of $f$, $g^{(k)}$, and $h_{k}$ implies that $\lim _{m \rightarrow \infty} f\left(t, x_{m}(t)\right)=f(t, x(t)), \lim _{m \rightarrow \infty} g^{(k)}\left(t, x_{m}(t)\right)=g^{(k)}(t, x(t))$, and $\lim _{m \rightarrow \infty} h_{k}\left(t, x_{m}(t)\right)=h_{k}(t, x(t))$. In this case, by the dominated convergence theorem,

$$
\begin{aligned}
\lim _{m \rightarrow \infty} & \Psi x_{m}(t) \\
= & I^{\alpha}\left(\lim _{m \rightarrow \infty} f\left(t, x_{m}(t)\right)\right)+\lim _{m \rightarrow \infty}\left(g\left(t, x_{m}(t)\right)-g\left(t_{0}, x_{m}\left(t_{0}\right)\right)\right) \\
& +\frac{\left(t-t_{0}\right) \Gamma\left(2-\rho_{0}\right)}{n_{0}\left(T-t_{0}\right)^{1-\rho_{0}}}\left(I^{\delta_{0}}\left(\lim _{m \rightarrow \infty} h_{0}\left(s, x_{m}(s)\right)\right)-\frac{m_{0}}{m_{1}} I^{\delta_{1}}\left(\lim _{m \rightarrow \infty} h_{1}\left(s, x_{m}(s)\right)\right)\right) \\
& +\frac{1}{m_{1}} I^{\delta_{1}}\left(\lim _{m \rightarrow \infty} h_{1}\left(s, x_{m}(s)\right)\right) \\
& +\frac{\left(t-t_{0}\right) \Gamma\left(2-\rho_{0}\right)}{\left(T-t_{0}\right)^{1-\rho_{0}}}\left(\frac{n_{1} m_{0}}{m_{1} n_{0}} I^{\alpha-\rho_{1}}\left(\lim _{m \rightarrow \infty} f\left(T, x_{m}(T)\right)\right)-I^{\alpha-\rho_{0}}\left(\lim _{m \rightarrow \infty} f\left(T, x_{m}(T)\right)\right)\right) \\
& -\frac{n_{1}}{m_{1}} I^{\alpha-\rho_{1}}\left(\lim _{m \rightarrow \infty} f\left(T, x_{m}(T)\right)\right) \\
& +\frac{\left(t-t_{0}\right) \Gamma\left(2-\rho_{0}\right)}{\left(T-t_{0}\right)^{1-\rho_{0}}}\left(\frac{n_{1} m_{0}}{m_{1} n_{0}} \lim _{m \rightarrow \infty} D^{\rho_{1}} g\left(T, x_{m}(T)\right)-\lim _{m \rightarrow \infty} D^{\rho_{0}} g\left(T, x_{m}(T)\right)\right) \\
& -\frac{n_{1}}{m_{1}} \lim _{m \rightarrow \infty} D^{\rho_{1}} g\left(T, x_{m}(T)\right) \\
& +\sum_{k=2}^{n-1}\left[\frac { n _ { 1 } ( T - t _ { 0 } ) ^ { k - \rho _ { 1 } } } { m _ { 1 } \Gamma ( k - \rho _ { 1 } + 1 ) } \left[1-\frac{m_{0}}{n_{0}} \frac{\left(t-t_{0}\right) \Gamma\left(2-\rho_{0}\right)}{\left.\left(T-t_{0}\right)^{1-\rho_{0}}\right]-\frac{\left(t-t_{0}\right)^{k}}{k !}}\right.\right.
\end{aligned}
$$




$$
\begin{aligned}
& \left.+\frac{\left(T-t_{0}\right)^{k-1}\left(t-t_{0}\right) \Gamma\left(2-\rho_{0}\right)}{\Gamma\left(k-\rho_{0}+1\right)}\right]\left[\lim _{m \rightarrow \infty} g^{(k)}\left(t_{0}, x_{m}\left(t_{0}\right)\right)-I^{\delta_{k}}\left(\lim _{m \rightarrow \infty} h_{k}\left(s, x_{m}(s)\right)\right)\right] \\
= & \Psi x(t), \quad t \in\left[t_{0}, T\right] .
\end{aligned}
$$

Thus, $\Psi$ is a continuous operator.

(Step 2) The operator $\Psi$ is uniformly bounded. $\forall t \in\left[t_{0}, T\right]$, we get

$$
\begin{aligned}
0 \leq & \Psi x(t) \\
\leq & \frac{\left(T-t_{0}\right)^{\alpha} L_{f}}{\Gamma(\alpha+1)}+L_{g} \\
& +\frac{\left(T-t_{0}\right) \Gamma\left(2-\rho_{0}\right)}{n_{0}\left(T-t_{0}\right)^{1-\rho_{0}}}\left(\frac{L_{h_{0}}\left(T-t_{0}\right)^{\delta_{0}}}{\Gamma\left(\delta_{0}+1\right)}+\frac{L_{h_{1}}\left(-m_{0}\right)\left(T-t_{0}\right)^{\delta_{1}}}{m_{1} \Gamma\left(\delta_{1}+1\right)}\right) \\
& +\frac{L_{h_{1}}\left(T-t_{0}\right)^{\delta_{1}}}{m_{1} \Gamma\left(\delta_{1}+1\right)}+\frac{L_{f}\left(T-t_{0}\right) \Gamma\left(2-\rho_{0}\right)}{\left(T-t_{0}\right)^{1-\rho_{0}}} \frac{\left(-n_{1}\right)\left(-m_{0}\right)\left(T-t_{0}\right)^{\alpha-\rho_{1}}}{m_{1} n_{0} \Gamma\left(\alpha-\rho_{1}+1\right)} \\
& +\frac{L_{f}\left(-n_{1}\right)\left(T-t_{0}\right)^{\alpha-\rho_{1}}}{m_{1} \Gamma\left(\alpha-\rho_{1}+1\right)}+\frac{\left(t-t_{0}\right) \Gamma\left(2-\rho_{0}\right)}{\left(T-t_{0}\right)^{1-\rho_{0}}} \frac{\left(-n_{1}\right)\left(-m_{0}\right) L_{g^{(2)}}\left(T-t_{0}\right)^{2-\rho_{1}}}{m_{1} n_{0} \Gamma\left(3-\rho_{1}\right)} \\
& +\frac{\left(-n_{1}\right) L_{g^{(2)}}\left(T-t_{0}\right)^{2-\rho_{1}}}{m_{1} \Gamma\left(3-\rho_{1}\right)} \\
& +\sum_{k=2}^{n-1}\left[\frac{\left(T-t_{0}\right)^{k}}{k !}-\frac{n_{1}\left(T-t_{0}\right)^{k-\rho_{1}}}{m_{1} \Gamma\left(k-\rho_{1}+1\right)}\left[1-\frac{m_{0}}{n_{0}} \frac{\left(t-t_{0}\right) \Gamma\left(2-\rho_{0}\right)}{\left(T-t_{0}\right)^{1-\rho_{0}}}\right]\right. \\
& \left.-\frac{\left(T-t_{0}\right)^{k-1}\left(t-t_{0}\right) \Gamma\left(2-\rho_{0}\right)}{\Gamma\left(k-\rho_{0}+1\right)}\right] \frac{L_{h_{k}}\left(T-t_{0}\right)^{\delta_{k}}}{\Gamma\left(\delta_{k}+1\right)} .
\end{aligned}
$$

Hence, $\Psi$ maps a bounded set $B_{r}$ into a uniformly bounded subset of $P$.

(Step 3) $\Psi B_{r}$ is an equicontinuous set in $P$. Let $x \in B_{r}$ and $t_{2}, t_{1} \in\left[t_{0}, T\right]$ such that $t_{1}<t_{2}$, then

$$
\begin{aligned}
&\left|\Psi x\left(t_{2}\right)-\Psi x\left(t_{1}\right)\right| \\
& \leq \frac{L_{f}}{\Gamma(\alpha)} \int_{t_{0}}^{t_{1}}\left[\left(t_{2}-s\right)^{\alpha-1}-\left(t_{1}-s\right)^{\alpha-1}\right] d s \\
&+\frac{L_{f}}{\Gamma(\alpha)} \int_{t_{1}}^{t_{2}}\left(t_{2}-s\right)^{\alpha-1} d s+\left|g\left(t_{2}, x\left(t_{2}\right)\right)-g\left(t_{1}, x\left(t_{1}\right)\right)\right| \\
&+\frac{\left(t_{2}-t_{1}\right) \Gamma\left(2-\rho_{0}\right)}{n_{0}\left(T-t_{0}\right)^{1-\rho_{0}}}\left|I^{\delta_{0}} h_{0}(T, x(T))-\frac{m_{0}}{m_{1}} I^{\delta_{1}} h_{1}(T, x(T))\right| \\
&+\frac{\left(t_{2}-t_{1}\right) \Gamma\left(2-\rho_{0}\right)}{\left(T-t_{0}\right)^{1-\rho_{0}}}\left|\frac{n_{1} m_{0}}{m_{1} n_{0}} I^{\alpha-\rho_{1}} f(T, x(T))-I^{\alpha-\rho_{0}} f(T, x(T))\right| \\
&+\frac{\left(t_{2}-t_{1}\right) \Gamma\left(2-\rho_{0}\right)}{\left(T-t_{0}\right)^{1-\rho_{0}}}\left|\frac{n_{1} m_{0}}{m_{1} n_{0}} D^{\rho_{1}} g(T, x(T))-D^{\rho_{0}} g(T, x(T))\right| \\
&+\sum_{k=2}^{n-1}\left[\frac{\left(t_{2}-t_{0}\right)^{k}-\left(t_{1}-t_{0}\right)^{k}}{k !}+\frac{m_{0} n_{1} \Gamma\left(2-\rho_{0}\right)\left(T-t_{0}\right)^{k+\rho_{0}-\rho_{1}-1}\left(t_{2}-t_{1}\right)}{n_{0} m_{1} \Gamma\left(k-\rho_{1}+1\right)}\right. \\
&\left.-\frac{\left(T-t_{0}\right)^{k-1}\left(t-t_{0}\right) \Gamma\left(2-\rho_{0}\right)\left(t_{2}-t_{1}\right)}{\Gamma\left(k-\rho_{0}+1\right)}\right]\left|I^{\delta_{k}} h_{k}(T, x(T))-g^{(k)}\left(t_{0}, x\left(t_{0}\right)\right)\right|
\end{aligned}
$$




$$
\begin{aligned}
\leq & L_{f} \frac{\left(t_{2}-t_{0}\right)^{\alpha}-\left(t_{1}-t_{0}\right)^{\alpha}+2\left(t_{2}-t_{1}\right)^{\alpha}}{\Gamma(\alpha+1)}+L_{r}\left|\mathfrak{g}\left(t_{2}\right)-\mathfrak{g}\left(t_{1}\right)\right| \\
& +\frac{\left(t_{2}-t_{1}\right) \Gamma\left(2-\rho_{0}\right)}{n_{0}\left(T-t_{0}\right)^{1-\rho_{0}}}\left(\frac{L_{h_{0}}\left(T-t_{0}\right)^{\delta_{0}}}{\Gamma\left(\delta_{0}+1\right)}+\frac{L_{h_{1}}\left|m_{0}\right|\left(T-t_{0}\right)^{\delta_{1}}}{m_{1} \Gamma\left(\delta_{1}+1\right)}\right) \\
& +\frac{L_{f}\left(t_{2}-t_{1}\right) \Gamma\left(2-\rho_{0}\right)}{\left(T-t_{0}\right)^{1-\rho_{0}}}\left(\frac{n_{1} m_{0}\left(T-t_{0}\right)^{\alpha-\rho_{1}}}{m_{1} n_{0} \Gamma\left(\alpha-\rho_{1}+1\right)}+\frac{\left(T-t_{0}\right)^{\alpha-\rho_{0}}}{\Gamma\left(\alpha-\rho_{0}+1\right)}\right) \\
& +\frac{\left(t_{2}-t_{1}\right) \Gamma\left(2-\rho_{0}\right)}{\left(T-t_{0}\right)^{1-\rho_{0}}}\left(\frac{n_{1} m_{0} L_{g^{(2)}}\left(T-t_{0}\right)^{2-\rho_{1}}}{m_{1} n_{0} \Gamma\left(3-\rho_{1}\right)}+\frac{L_{g^{(1)}}\left(T-t_{0}\right)^{1-\rho_{0}}}{\Gamma\left(2-\rho_{0}\right)}\right) \\
& +\sum_{k=2}^{n-1}\left[\frac{\left(t_{2}-t_{0}\right)^{k}-\left(t_{1}-t_{0}\right)^{k}}{k !}+\frac{m_{0} n_{1} \Gamma\left(2-\rho_{0}\right)\left(T-t_{0}\right)^{k+\rho_{0}-\rho_{1}-1}\left(t_{2}-t_{1}\right)}{n_{0} m_{1} \Gamma\left(k-\rho_{1}+1\right)}\right. \\
& \left.-\frac{\left(T-t_{0}\right)^{k-1}\left(t-t_{0}\right) \Gamma\left(2-\rho_{0}\right)\left(t_{2}-t_{1}\right)}{\Gamma\left(k-\rho_{0}+1\right)}\right]\left|\frac{L_{h_{k}}\left(T-t_{0}\right)^{\delta_{k}}}{\Gamma\left(\delta_{k}+1\right)}+L_{g^{(k)}}\right|
\end{aligned}
$$

If $t_{2}-t_{1} \rightarrow 0$, then $\left|\Psi x\left(t_{2}\right)-\Psi x\left(t_{1}\right)\right| \rightarrow 0$ independently of the values of $x$. Hence, $\Psi B_{r}$ is equicontinuous. By the means of the Arzela-Ascoli theorem, we follow that $\Psi: P \rightarrow P$ is completely continuous.

We now show the existence of at least two solutions for the hybrid FBVP (1).

For simplifications, we use the following notations in the coming results:

$$
\begin{aligned}
\left|\xi_{k}(t)\right| & \leq M_{\xi_{k}}, \\
R_{1}= & m_{f}\left(T-t_{0}\right)^{\alpha}\left(\frac{1}{\Gamma(\alpha+1)}+\frac{\left|n_{1}\right|}{m_{1}\left(T-t_{0}\right)^{\rho_{1}} \Gamma\left(\alpha-\rho_{1}+1\right)}\right) \\
& +\frac{m_{h_{0}}\left(T-t_{0}\right)^{\delta_{0}+\rho_{0}}}{n_{0}} \frac{\Gamma\left(2-\rho_{0}\right)}{\Gamma\left(\delta_{0}+1\right)}+\frac{m_{h_{1}}\left(T-t_{0}\right)^{\delta_{1}}}{m_{1} \Gamma\left(\delta_{1}+1\right)}\left(1+\frac{\left|m_{0}\right|}{n_{0}}\left(T-t_{0}\right)^{\rho_{0}} \Gamma\left(2-\rho_{0}\right)\right) \\
R_{2}= & M_{f}\left(T-t_{0}\right)^{\alpha}\left(\frac{1}{\Gamma(\alpha+1)}+\frac{\left|n_{1}\right|\left(1+\frac{m_{0}\left(T-t_{0}\right)^{\rho_{0}} \Gamma\left(2-\rho_{0}\right)}{n_{0}}\right)}{m_{1}\left(T-t_{0}\right)^{\rho_{1}} \Gamma\left(\alpha-\rho_{1}+1\right)}+\frac{\Gamma\left(2-\rho_{0}\right)}{\Gamma\left(\alpha-\rho_{0}+1\right)}\right) \\
& +M_{g}\left(2+K\left(\frac{n_{0}}{\left|m_{0}\right|}+\frac{n_{1} m_{0}}{m_{1} n_{0}}\left(T-t_{0}\right)^{\rho_{0}} \Gamma\left(2-\rho_{0}\right)\right)\right) \\
& +\frac{M_{h_{0}}\left(T-t_{0}\right)^{\delta_{0}+\rho_{0}} \Gamma\left(2-\rho_{0}\right)}{n_{0} \Gamma\left(\delta_{0}+1\right)}+\frac{M_{h_{1}}\left(T-t_{0}\right)^{\delta_{1}}}{m_{1} \Gamma\left(\delta_{1}+1\right)}\left(1+\frac{\left|m_{0}\right|\left(T-t_{0}\right)^{\rho_{0}} \Gamma\left(2-\rho_{0}\right)}{n_{0}}\right) \\
& +2 \sum_{k=2}^{n-1} M_{\xi_{k}} M_{h_{k}}\left(1+\frac{\left(T-t_{0}\right)^{\delta_{k}}}{\Gamma\left(\delta_{k}+1\right)}\right)
\end{aligned}
$$

and

$$
\begin{aligned}
R_{3}= & \frac{n_{f}\left(\tau-t_{0}\right)^{\alpha}}{\Gamma(\alpha+1)}+\frac{n_{h_{1}}\left(T-t_{0}\right)^{\delta_{1}}}{m_{1} \Gamma\left(\delta_{1}+1\right)} \\
& +\frac{\left(\tau-t_{0}\right) \Gamma\left(2-\rho_{0}\right)}{n_{0}\left(T-t_{0}\right)^{1-\rho_{0}}}\left(\frac{n_{h_{0}}\left(T-t_{0}\right)^{\delta_{0}}}{\Gamma\left(\delta_{0}+1\right)}+\frac{\left|m_{0}\right| n_{h_{1}}\left(T-t_{0}\right)^{\delta_{1}}}{m_{1} \Gamma\left(\delta_{1}+1\right)}\right) \\
& +\frac{\left|n_{1}\right|\left(T-t_{0}\right)^{\alpha-\rho_{1}} n_{f}}{m_{1} \Gamma\left(\alpha-\rho_{1}+1\right)}, \quad \tau \in\left[t_{0}, T\right],
\end{aligned}
$$

where the involved constants exist and are positive. 
Theorem 4.3 Let [H2] and [H3] hold. If there exist $0<r_{1}<r_{2}<r_{3}$ satisfying

(i) $f(t, x)>n_{f} \frac{r_{3}}{R_{3}}, h_{i}(t, x)>n_{h_{i}} \frac{r_{3}}{R_{3}}, i=0,1, t \in[\tau, T], x \geq r_{3}$,

(ii) $f(t, x) \leq M_{f} \frac{r_{2}}{R_{2}}, h_{i}(t, x) \leq M_{h_{k}} \frac{r_{2}}{R_{2}}, i=0,1,(t, x) \in\left[t_{0}, T\right] \times\left[0, r_{2}\right]$, and

(iii) $f(t, x)>m_{f} \frac{r_{1}}{R_{1}}, h_{i}(t, x)>m_{h_{i}} \frac{r_{1}}{R_{1}}, i=0,1,(t, x) \in\left[t_{0}, T\right] \times\left[0, r_{1}\right]$,

then hybrid system (1) possesses at least two positive solutions $x_{1}$ and $x_{2}$ provided that $r_{1}<\left\|x_{1}\right\|$ with $\left\|x_{1}\right\|<r_{2}$ along with $r_{2}<\left\|x_{2}\right\|$ with $\min _{t \in[\tau, T]} x(t)<r_{3}$.

Proof Let $\tau \in\left[t_{0}, T\right)$ and define the functionals $\phi$, $\omega$, and $\mu$ on the cone $P$ such that

$$
\phi(x)=\min _{t \in[\tau, T]} x(t), \quad \omega(x)=\mu(x)=\|x\| .
$$

It is obvious that $\omega(0)=0, \omega(l x)=|l| \omega(x)$, and $\phi(x) \leq \omega(x) \leq \mu(x)$. Using Lemmas 4.1 and 4.2, we have $\Psi: \overline{P\left(\phi, r_{3}\right)} \rightarrow P$ is completely continuous. We start with the first condition of Theorem 2.5, namely $\phi(\Psi x)>r_{3}$ for all $x \in \partial P\left(\phi, r_{3}\right)$. Let $\min _{t \in[\tau, T]} x(t)=r_{3}$, we get

$$
\begin{aligned}
\min _{t \in[\tau, T]} \Psi x(t)> & \frac{n_{f} r_{3}}{R_{3}} \frac{\left(\tau-t_{0}\right)^{\alpha}}{\Gamma(\alpha+1)}+\frac{n_{h_{1}} r_{3}\left(T-t_{0}\right)^{\delta_{1}}}{m_{1} R_{3} \Gamma\left(\delta_{1}+1\right)} \\
& +\frac{\left(\tau-t_{0}\right) \Gamma\left(2-\rho_{0}\right)}{n_{0}\left(T-t_{0}\right)^{1-\rho_{0}}}\left(\frac{n_{h_{0}} r_{3}\left(T-t_{0}\right)^{\delta_{0}}}{R_{3} \Gamma\left(\delta_{0}+1\right)}+\frac{\left|m_{0}\right| n_{h_{1}} r_{3}\left(T-t_{0}\right)^{\delta_{1}}}{m_{1} R_{3} \Gamma\left(\delta_{1}+1\right)}\right) \\
& +\frac{\left|n_{1}\right|\left(T-t_{0}\right)^{\alpha-\rho_{1}} n_{f} r_{3}}{m_{1} \Gamma\left(\alpha-\rho_{1}+1\right) R_{3}} \\
= & r_{3} .
\end{aligned}
$$

Next, we check the second condition. Let $x \in \partial P\left(\omega, r_{2}\right)$, then

$$
\begin{aligned}
\Psi x(t) \leq & \frac{\left(T-t_{0}\right)^{\alpha} M_{f} r_{2}}{\Gamma(\alpha+1) R_{2}}+\frac{2 M_{g} r_{2}}{R_{2}}+\frac{M_{h_{1}} r_{2}\left(T-t_{0}\right)^{\delta_{1}}}{m_{1} R_{2} \Gamma\left(\delta_{1}+1\right)} \\
& +\frac{\left(T-t_{0}\right)^{\rho_{0}} \Gamma\left(2-\rho_{0}\right) r_{2}}{n_{0} R_{2}}\left(\frac{M_{h_{0}}\left(T-t_{0}\right)^{\delta_{0}}}{\Gamma\left(\delta_{0}+1\right)}+\frac{\left|m_{0}\right| M_{h_{1}}\left(T-t_{0}\right)^{\delta_{1}}}{m_{1} \Gamma\left(\delta_{1}+1\right)}\right) \\
& +\frac{M_{f}\left(T-t_{0}\right)^{\rho_{0}} \Gamma\left(2-\rho_{0}\right) r_{2}}{R_{2}}\left(\frac{n_{1} m_{0}\left(T-t_{0}\right)^{\alpha-\rho_{1}}}{m_{1} n_{0} \Gamma\left(\alpha-\rho_{1}+1\right)}+\frac{\left(T-t_{0}\right)^{\alpha-\rho_{0}}}{\Gamma\left(\alpha-\rho_{0}+1\right)}\right) \\
& +\frac{M_{f}\left|n_{1}\right|\left(T-t_{0}\right)^{\alpha-\rho_{1}} r_{2}}{m_{1} \Gamma\left(\alpha-\rho_{1}+1\right) R_{2}}+\frac{r_{2} n_{0} K M_{g}}{\left|m_{0}\right| R_{2}} \\
& +\frac{\left(T-t_{0}\right)^{\rho_{0}} \Gamma\left(2-\rho_{0}\right)}{R_{2}} \frac{n_{1} m_{0} K M_{g} r_{2}}{m_{1} n_{0}} \\
& +\frac{2 r_{2}}{R_{2}} \sum_{k=2}^{n-1} M_{\xi_{k}} M_{h_{k}}\left(1+\frac{\left(T-t_{0}\right)^{\delta_{k}}}{\Gamma\left(\delta_{k}+1\right)}\right) .
\end{aligned}
$$

This shows that $\omega(\Psi x)=\|\Psi x\|<r_{2}$. Since $0 \in P$ and $r_{1}>0$, hence $P\left(\mu, r_{1}\right) \neq \emptyset$. By assuming $x \in \partial P\left(\mu, r_{1}\right)$, we have $0 \leq x(t) \leq r_{1}, \forall t \in\left[t_{0}, T\right]$. By assumption (iii), we have

$$
\begin{aligned}
\mu(\Psi x) & =\max _{t \in\left[t_{0}, T\right]} \Psi x(t) \\
& >\frac{m_{f} r_{1}}{R_{1}} \frac{\left(T-t_{0}\right)^{\alpha}}{\Gamma(\alpha+1)}+\frac{m_{h_{1}} r_{1}\left(T-t_{0}\right)^{\delta_{1}}}{m_{1} R_{1} \Gamma\left(\delta_{1}+1\right)}
\end{aligned}
$$




$$
\begin{aligned}
& +\frac{\left(T-t_{0}\right)^{\rho_{0}} \Gamma\left(2-\rho_{0}\right)}{n_{0}}\left(\frac{m_{h_{0}} r_{1}\left(T-t_{0}\right)^{\delta_{0}}}{R_{1} \Gamma\left(\delta_{0}+1\right)}+\frac{\left|m_{0}\right| m_{h_{1}} r_{1}\left(T-t_{0}\right)^{\delta_{1}}}{m_{1} R_{1} \Gamma\left(\delta_{1}+1\right)}\right) \\
& +\frac{\left|n_{1}\right|\left(T-t_{0}\right)^{\alpha-\rho_{1}} m_{f} r_{1}}{m_{1} \Gamma\left(\alpha-\rho_{1}+1\right) R_{1}} .
\end{aligned}
$$

Hence, $\mu(\Psi x)>r_{1}$. All the conditions of Theorem 2.5 are established, and the desired result follows.

\section{Hyers-Ulam stability}

The notion of the stability of functional differential equations was first introduced by Ulam [50], and then it was extended by Hyers [51]. Later on, this type of stability and its generalization were called of Hyers-Ulam (HU) and Hyers-Ulam-Rassias (HUR) type, respectively. Investigation of the $\mathrm{UH}$ and GUH stability has been given a special attention in studying all fractional differential equations. Here, we discuss the Hyers-Ulam (HU) and Hyers-Ulam-Rassias (HUR) stability results about the hybrid FBVP (1) on the interval $\left[t_{0}, T\right]$.

Definition 5.1 System (1) is Hyers-Ulam stable whenever for every $\epsilon>0$ and $y \epsilon$ $C\left(\left[t_{0}, T\right], \mathbb{R}\right)$ satisfying

$$
\left|D^{\alpha}[y(t)-g(t, y(t))]-f(t, y(t))\right| \leq \epsilon, \quad t \in\left[t_{0}, T\right], \alpha \in(n-1, n),
$$

there exists $x(t)$ as a solution of (1) such that

$$
|x(t)-y(t)| \leq C \epsilon, \quad t \in\left[t_{0}, T\right]
$$

where $C$ is independent of both $y$ and $x$.

Definition 5.2 System (1) is Hyers-Ulam-Rassias stable if $\forall y \in C\left(\left[t_{0}, T\right], \mathbb{R}\right)$ satisfying

$$
\left|D^{\alpha}[y(t)-g(t, y(t))]-f(t, y(t))\right| \leq \varphi(t), \quad t \in\left[t_{0}, T\right],
$$

where $\varphi:\left[t_{0}, T\right] \rightarrow \mathbb{R}$ is continuous, there is $x(t)$ as a solution of $(1)$, provided

$$
|x(t)-y(t)| \leq C \varphi(t), \quad t \in\left[t_{0}, T\right]
$$

where $C$ is independent of both $y$ and $x$.

For simplification, set

$$
\begin{aligned}
& \Theta(y, f(t, y(t))) \\
& =I^{\alpha} f(t, y(t))+g(t, y(t))-g\left(t_{0}, y\left(t_{0}\right)\right) \\
& \quad+\frac{\left(t-t_{0}\right) \Gamma\left(2-\rho_{0}\right)}{n_{0}\left(T-t_{0}\right)^{1-\rho_{0}}}\left(I^{\delta_{0}} h_{0}(T, x(T))-\frac{m_{0}}{m_{1}} I^{\delta_{1}} h_{1}(T, y(T))\right)+\frac{1}{m_{1}} I^{\delta_{1}} h_{1}(T, y(T)) \\
& \quad+\frac{\left(t-t_{0}\right) \Gamma\left(2-\rho_{0}\right)}{\left(T-t_{0}\right)^{1-\rho_{0}}}\left(\frac{n_{1} m_{0}}{m_{1} n_{0}} I^{\alpha-\rho_{1}} f(T, y(T))-I^{\alpha-\rho_{0}} f(T, y(T))\right)
\end{aligned}
$$




$$
\begin{aligned}
& -\frac{n_{1}}{m_{1}} I^{\alpha-\rho_{1}} f(T, y(T)) \\
& +\frac{\left(t-t_{0}\right) \Gamma\left(2-\rho_{0}\right)}{\left(T-t_{0}\right)^{1-\rho_{0}}}\left(\frac{n_{1} m_{0}}{m_{1} n_{0}} D^{\rho_{1}} g(T, y(T))-D^{\rho_{0}} g(T, y(T))\right)-\frac{n_{1}}{m_{1}} D^{\rho_{1}} g(T, y(T)) \\
& +\sum_{k=2}^{n-1}\left[\frac{n_{1}\left(T-t_{0}\right)^{k-\rho_{1}}}{m_{1} \Gamma\left(k-\rho_{1}+1\right)}\left[1-\frac{m_{0}}{n_{0}} \frac{\left(t-t_{0}\right) \Gamma\left(2-\rho_{0}\right)}{\left(T-t_{0}\right)^{1-\rho_{0}}}\right]-\frac{\left(t-t_{0}\right)^{k}}{k !}\right. \\
& \left.+\frac{\left(T-t_{0}\right)^{k-1}\left(t-t_{0}\right) \Gamma\left(2-\rho_{0}\right)}{\Gamma\left(k-\rho_{0}+1\right)}\right]\left[g^{(k)}\left(t_{0}, y\left(t_{0}\right)\right)-I^{\delta_{k}} h_{k}(T, y(T))\right] .
\end{aligned}
$$

Remark 5.1 $y \in C\left(\left[t_{0}, T\right], \mathbb{R}\right)$ is a solution of (13) iff we can find $\hbar \in C\left(\left[t_{0}, T\right], \mathbb{R}\right)$ so that

(1) $|\hbar(t)| \leq \epsilon, t \in\left[t_{0}, T\right]$;

(2) $y$ satisfies the equation

$$
y(t)=\Theta(y, \hbar(t)+f(t, y(t)))=\Theta(y, f(t, y(t)))+I^{\alpha} \hbar(t) .
$$

A similar remark can be obtained on considering inequality (14).

Lemma 5.3 A function $y \in C\left(\left[t_{0}, T\right], \mathbb{R}\right)$ satisfying (13) also satisfies the following integral inequality:

$$
|y(t)-\Theta(y, f(t, y(t)))| \leq \frac{\left(T-t_{0}\right)^{\alpha}}{\Gamma(\alpha+1)} \epsilon .
$$

Proof According to Remark 5.1, $y$ satisfies equation (15). As a result,

$$
\begin{aligned}
|y(t)-\Theta(y, f(t, y(t)))| & =\left|I^{\alpha} \hbar(t)\right| \\
& \leq \frac{1}{\Gamma(\alpha)} \int_{t_{0}}^{t}(t-s)^{\alpha-1}|\hbar(s)| d s \\
& \leq \frac{\left(T-t_{0}\right)^{\alpha}}{\Gamma(\alpha+1)} \epsilon .
\end{aligned}
$$

Theorem 5.4 If $[\mathrm{H} 1]$ is fulfilled, hybrid system (1) is Hyers-Ulam stable, provided that $\Delta<1$.

Proof Take $\epsilon>0$ and $y \in C\left(\left[t_{0}, T\right], \mathbb{R}\right)$ satisfying (13), and let $x \in C\left(\left[t_{0}, T\right], \mathbb{R}\right)$ be the unique solution of (1). Thus,

$$
\begin{aligned}
\mid y(t) & -x(t) \mid \\
\leq & \frac{1}{\Gamma(\alpha)} \int_{t_{0}}^{t}(t-s)^{\alpha-1}|f(s, y(s))-f(s, x(s))| d s+|g(t, y(t))-g(t, x(t))| \\
& +\left|g\left(t_{0}, y\left(t_{0}\right)\right)-g\left(t_{0}, x\left(t_{0}\right)\right)\right| \\
& +\frac{\left(t-t_{0}\right) \Gamma\left(2-\rho_{0}\right)}{n_{0}\left(T-t_{0}\right)^{1-\rho_{0}}}\left[\frac{1}{\Gamma\left(\delta_{0}\right)} \int_{t_{0}}^{T}(T-s)^{\delta_{0}-1}\left|h_{0}(s, y(s))-h_{0}(s, x(s))\right| d s\right.
\end{aligned}
$$




$$
\begin{aligned}
& \left.-\frac{m_{0}}{m_{1} \Gamma\left(\delta_{1}\right)} \int_{t_{0}}^{T}(T-s)^{\delta_{1}-1}\left|h_{1}(s, y(s))-h_{1}(s, x(s))\right| d s\right] \\
& +\frac{1}{m_{1} \Gamma\left(\delta_{1}\right)} \int_{t_{0}}^{T}(T-s)^{\delta_{1}-1}\left|h_{1}(s, y(s))-h_{1}(s, x(s))\right| d s \\
& +\frac{\left(t-t_{0}\right) \Gamma\left(2-\rho_{0}\right)}{\left(T-t_{0}\right)^{1-\rho_{0}}}\left[\frac{n_{1} m_{0}}{m_{1} n_{0} \Gamma\left(\alpha-\rho_{1}\right)} \int_{t_{0}}^{T}(T-s)^{\alpha-\rho_{1}-1}|f(s, y(s))-f(s, x(s))| d s\right. \\
& \left.+\frac{1}{\Gamma\left(\alpha-\rho_{0}\right)} \int_{t_{0}}^{T}(T-s)^{\alpha-\rho_{0}-1}|f(s, y(s))-f(s, x(s))| d s\right] \\
& -\frac{n_{1}}{m_{1} \Gamma\left(\alpha-\rho_{1}\right)} \int_{t_{0}}^{T}(T-s)^{\alpha-\rho_{1}-1}|f(s, y(s))-f(s, x(s))| d s \\
& +\frac{\left(t-t_{0}\right) \Gamma\left(2-\rho_{0}\right)}{\left(T-t_{0}\right)^{1-\rho_{0}}}\left[\frac{n_{1} m_{0}}{m_{1} n_{0} \Gamma\left(2-\rho_{1}\right)} \int_{t_{0}}^{T}(T-s)^{1-\rho_{1}}\left|g^{\prime \prime}(s, y(s))-g^{\prime \prime}(s, x(s))\right| d s\right. \\
& \left.+\frac{1}{\Gamma\left(1-\rho_{0}\right)} \int_{t_{0}}^{T}(T-s)^{-\rho_{0}}\left|g^{\prime}(s, y(s))-g^{\prime}(s, x(s))\right| d s\right] \\
& -\frac{n_{1}}{m_{1} \Gamma\left(2-\rho_{1}\right)} \int_{t_{0}}^{T}(T-s)^{1-\rho_{1}}\left|g^{\prime \prime}(s, y(s))-g^{\prime \prime}(s, x(s))\right| d s \\
& +\sum_{k=2}^{n-1}\left|\xi_{k}(t)\right|\left|g^{(k)}\left(t_{0}, x\left(t_{0}\right)\right)-g^{(k)}\left(t_{0}, y\left(t_{0}\right)\right)\right| \\
& +\frac{1}{\Gamma\left(\delta_{k}\right)} \int_{t_{0}}^{T}(T-s)^{\delta_{k}-1}\left|h_{k}(s, y(s))-h_{k}(s, x(s))\right| d s+\frac{\left(T-t_{0}\right)^{\alpha}}{\Gamma(\alpha+1)} \epsilon \\
& \leq \frac{C_{f}\left(T-t_{0}\right)^{\alpha}}{\Gamma(\alpha+1)}\|y-x\|+2 C_{g}\|y-x\| \\
& +\frac{\left(T-t_{0}\right)^{\rho_{0}} \Gamma\left(2-\rho_{0}\right)}{n_{0}}\left[\frac{\left(T-t_{0}\right)^{\delta_{0}} C_{h_{0}}}{\Gamma\left(\delta_{0}+1\right)}-\frac{m_{0}\left(T-t_{0}\right)^{\delta_{1}} C_{h_{1}}}{m_{1} \Gamma\left(\delta_{1}+1\right)}\right]\|y-x\| \\
& -\frac{\left(T-t_{0}\right)^{\delta_{1}} C_{h_{1}}}{m_{1} \Gamma\left(\delta_{1}+1\right)}\|y-x\| \\
& +\left(T-t_{0}\right)^{\rho_{0}} \Gamma\left(2-\rho_{0}\right) C_{f}\left[\frac{n_{1} m_{0}\left(T-t_{0}\right)^{\alpha-\rho_{1}}}{m_{1} n_{0} \Gamma\left(\alpha-\rho_{1}+1\right)}+\frac{\left(T-t_{0}\right)^{\alpha-\rho_{0}}}{\Gamma\left(\alpha-\rho_{0}+1\right)}\right]\|y-x\| \\
& -\frac{n_{1}\left(T-t_{0}\right)^{\alpha-\rho_{1}} C_{f}}{m_{1} \Gamma\left(\alpha-\rho_{1}+1\right)}\|y-x\| \\
& +\left(T-t_{0}\right)^{\rho_{1}} \Gamma\left(2-\rho_{0}\right)\left[\frac{n_{1} m_{0} C_{g^{(2)}}\left(T-t_{0}\right)^{2-\rho_{1}}}{m_{1} n_{0} \Gamma\left(3-\rho_{1}\right)}+\frac{\left(T-t_{0}\right)^{1-\rho_{0}} C_{g^{(1)}}}{\Gamma\left(2-\rho_{0}\right)}\right]\|y-x\| \\
& -\frac{n_{1} C_{g^{(2)}}\left(T-t_{0}\right)^{2-\rho_{1}}}{m_{1} \Gamma\left(3-\rho_{1}\right)}\|y-x\| \\
& +\sum_{k=2}^{n-1} M_{\xi_{k}}\left[C_{g^{(k)}}+\frac{\left(T-t_{0}\right)^{\delta_{k}} C_{h_{k}}}{\Gamma\left(\delta_{k}+1\right)}\right]\|y-x\|+\frac{\left(T-t_{0}\right)^{\alpha}}{\Gamma(\alpha+1)} \epsilon \\
& =\Delta\|y-x\|+\frac{\left(T-t_{0}\right)^{\alpha}}{\Gamma(\alpha+1)} \epsilon \text {. }
\end{aligned}
$$

Hence,

$$
\|y-x\| \leq \frac{\left(T-t_{0}\right)^{\alpha}}{(1-\Delta) \Gamma(\alpha+1)} \epsilon:=C \epsilon,
$$


where

$$
C=\frac{\left(T-t_{0}\right)^{\alpha}}{(1-\Delta) \Gamma(\alpha+1)}
$$

Hence, system (1) is Hyers-Ulam stable.

Remark 5.2 The Hyers-Ulam-Rassias stability can be established in a similar manner.

\section{Example}

In this portion, we give an example to defend our pivot results of the theory attained above.

Example 6.1 Due to (1), regard the following fractional hybrid differential system:

$$
\left\{\begin{array}{l}
D^{2.5}(x(t)-g(t, x(t)))=f(t, x(t)), \quad t \in(0,1) \\
-x(0)+D^{0.5} x(1)=I^{2} h_{0}(1, x(1)) \\
x(0)-D^{1.5} x(1)=I^{2} h_{1}(1, x(1)) \\
x^{(2)}(0)=I^{2} h_{2}(1, x(1))
\end{array}\right.
$$

where $\alpha=2.5, \rho_{0}=0.5, \rho_{1}=1.5$, and $\delta_{0}=\delta_{1}=\delta_{2}=2$. Since the functions $f, h_{k}, g^{(k)}, k=$ $0,1,2$, are Lipschitz with $C_{f}=C_{h_{k}}=C_{g^{(k)}}=0.01$. Therefore, we find that

$$
\Delta=0.126<1 .
$$

Hence, problem (16) has a unique solution by Theorem 3.3. Moreover, using Theorem 5.4, system (16) is Hyers-Ulam (-Rassias) stable with $C=2.39$.

Let us, in particular, assume that

$$
\begin{aligned}
& f(t, x)=h_{0}(t, x)=h_{1}(t, x)= \begin{cases}0.4, & x \leq 1, \\
0.39+\frac{x}{100}, & 1 \leq x \leq 5, \\
2.56 x-12.36, & 5 \leq x \leq 6, \\
3, & x \geq 6,\end{cases} \\
& g(t, x)=0.1 e^{t}, \quad h_{2}(t, x)=e^{t} .
\end{aligned}
$$

One can find figures of the functions $f(t, x)$ and $g(t, x)$ in [52]. The calculations of the basic conditions give the following:

$$
\left\{\begin{array}{l}
m_{0}, n_{1} \leq 0, \quad m_{1}, n_{0}>0, \\
\frac{\Gamma\left(3-\rho_{1}\right)}{\Gamma\left(3-\rho_{0}\right)}=0.667<1=\frac{n_{1} m_{0}}{m_{1} n_{0}}\left(T-t_{0}\right)^{\rho_{0}-\rho_{1}}, \\
f(t, x) \geq 0 \\
g(t, x(t)) \geq g\left(t_{0}, x\left(t_{0}\right)\right)=0.1 \geq 0, \quad t \in[0,1], \\
\frac{n_{1} m_{0}}{m_{1} n_{0}} I^{\alpha-\rho_{1}} f(T, x(T))=I^{1} f\left(1, x(1) \geq I^{2} f(1, x(1)),\right. \\
g(0.83, x(0.83)) \leq \frac{n_{1} m_{0}}{m_{1} n_{0}} D^{\rho_{1}} g(T, x(T))=D^{1.5} g(T, x(T)) \\
\quad=I^{0.5} g^{\prime \prime}(1, x(1))=I^{0.5} g^{\prime}(1, x(1))=D^{0.5} g(T, x(T)) \geq 0, \\
0 \leq g^{\prime \prime}(0, x(0))=0.1 \leq 0.7183=I^{\delta_{2}} h_{2}(1, x(1)) .
\end{array}\right.
$$


Moreover, it is obvious that condition [H3] is also valid. To continue our investigation, we need to justify the conditions of Theorem 4.3. Let $r_{1}=1, r_{2}=5$, and $r_{3}=6$. Also assume that

$$
\begin{aligned}
\tau= & 0.5, \\
m_{f} & =m_{h_{0}}=m_{h_{1}}=M_{f}=M_{g}=M_{h_{0}}=M_{h_{1}} \\
& =M_{h_{2}}=n_{f}=n_{h_{1}}=n_{h_{0}}=1 .
\end{aligned}
$$

Then we find that

$$
\left\{\begin{array}{l}
\left|\xi_{2}(t)\right| \leq M_{\xi_{2}}=0.89327 \\
R_{1}=1.3 m_{f}+0.443 m_{h_{0}}+0.943 m_{h_{1}}=2.686 \\
R_{2}=2.63 M_{f}+(2+1.88623 K) M_{g}+0.443 M_{h_{0}}+0.943 M_{h_{1}}+2.68 M_{h_{2}}=10.6 \\
R_{3}=1.0532 n_{f}+0.722 n_{h_{1}}+0.222 n_{h_{0}}=1.997
\end{array}\right.
$$

Therefore, hypotheses (i)-(iii) are satisfied, since if $t \in[0.5,1]$ and $x \geq 6$, we have

$$
\left\{\begin{array}{l}
f(t, x)>n_{f} \frac{r_{3}}{R_{3}}=3, \\
h_{k}(t, x)>n_{h_{k}} \frac{r_{3}}{R_{3}}=3, \quad k=0,1 .
\end{array}\right.
$$

For $(t, x) \in[0,1] \times[0,5]$, we obtain

$$
\left\{\begin{array}{l}
f(t, x) \leq M_{f} \frac{r_{2}}{R_{2}} \leq 0.472 \\
h_{k}(t, x) \leq M_{h_{k}} \frac{r_{2}}{R_{2}} \leq 0.472, \quad k=0,1
\end{array}\right.
$$

Finally, $\forall(t, x) \in[0,1] \times[0,1]$, we get

$$
\left\{\begin{array}{l}
f(t, x)>m_{f} \frac{r_{1}}{R_{1}}>0.3723 \\
h_{k}(t, x)>m_{h_{k}} \frac{r_{1}}{R_{1}}>0.3723, \quad k=0,1
\end{array}\right.
$$

Therefore, using Theorem 4.3, for system (16), the existence of at least two positive solutions $x_{1}$ and $x_{2}$ is guaranteed provided $1<\left\|x_{1}\right\|$ with $\left\|x_{1}\right\|<5$ and $5<\left\|x_{2}\right\|$ with $\min _{t \in[0.5,1]} x(t)<6$.

\section{Conclusion}

The fractional integro-differential boundary problem of a hybrid system is a generalization of many existing problems. Many basic expressions are gathered in this model such as hybrid model, fractional derivatives of any order, fractional intro-differential boundary conditions, etc. Based on some well-known fixed point theorems of operator theory and the technique of fractional nonlinear differential systems, the existence and uniqueness criteria for the considered system (1) have been obtained. To do this, we used some notions on cones and verified some inequalities. Likewise, under specific assumptions and conditions, we have found the Hyers-Ulam stability result regarding solutions of hybrid system (1). The future research may continue to develop many qualitative properties of 
a modified system with the very recent fractional derivatives containing nonsingular kernels.

\author{
Acknowledgements \\ The fifth and sixth authors would like to thank Azarbaijan Shahid Madani University. The authors would like to thank dear \\ referees for their constructive and valuable comments.
}

\title{
Funding
}

Not applicable.

\section{Availability of data and materials}

Data sharing not applicable to this article as no datasets were generated or analyzed during the current study.

\section{Ethics approval and consent to participate}

Not applicable.

\section{Competing interests}

The authors declare that they have no competing interests.

\section{Consent for publication}

Not applicable.

\section{Authors' contributions}

The authors declare that the study was realized in collaboration with equal responsibility. All authors read and approved the final manuscript.

\section{Author details}

'Department of Mathematics, Al-Azhar University-Gaza, Gaza Strip, State of Palestine. ${ }^{2}$ Department of Mathematics, University of Peshawar, Peshawar 25000, Pakistan. ${ }^{3}$ Department of Mathematics, Azarbaijan Shahid Madani University, Tabriz, Iran. ${ }^{4}$ Department of Medical Research, China Medical University Hospital, China Medical University, Taichung, Taiwan.

\section{Publisher's Note}

Springer Nature remains neutral with regard to jurisdictional claims in published maps and institutional affiliations.

Received: 23 August 2021 Accepted: 7 September 2021 Published online: 22 September 2021

\section{References}

1. Herrmann, R.: Fractional Calculus. An Introduction for Physicists. World Scientific, Singapore (2011)

2. Lakshmikantham, V., Leela, S., Vasundhara, D.J.: Theory of Fractional Dynamic Systems. Cambridge Academic Publishers, London (2009)

3. Sun, H., Zhang, Y., Baleanu, D., Chen, W., Chen, Y.: A new collection of real world applications of fractional calculus in science and engineering. Commun. Nonlinear Sci. Numer. Simul. 64, 213-231 (2018). https://doi.org/10.1016/j.cnsns.2018.04.019

4. Ahmad, M., Zada, A., Alzabut, J.: Hyers-Ulam stability of a coupled system of fractional differential equations of Hilfer-Hadamard type. Demonstr. Math. 52(1), 283-295 (2019). https://doi.org/10.1515/dema-2019-0024

5. Ahmad, M., Zada, A., Alzabut, J.: Stability analysis of a nonlinear coupled implicit switched singular fractional differential system with p-Laplacian. Adv. Differ. Equ. 2019, 436 (2019). https://doi.org/10.1186/s13662-019-2367-y

6. Riaz, U., Zada, A., Ali, Z., Ahmad, M., Xu, J., Fu, Z.: Analysis of nonlinear coupled systems of impulsive fractional differential equations with Hadamard derivatives. Math. Probl. Eng. 2019, Article ID 5093572 (2019). https://doi.org/10.1155/2019/5093572

7. Baleanu, D., Aydogan, S.M., Mohammadi, H., Rezapour, S.: On modelling of epidemic childhood diseases with the Caputo-Fabrizio derivative by using the Laplace Adomian decomposition method. Alex. Eng. J. 59(5), 3029-3039 (2020). https://doi.org/10.1016/j.aej.2020.05.007

8. Bachir, F.S., Abbas, S., Benbachir, M., Benchora, M.: Hilfer-Hadamard fractional differential equations; existence and attractivity. Adv. Theory Nonlinear Anal. Appl. 5(1), 49-57 (2021). https://doi.org/10.31197/atnaa.848928

9. Baitiche, Z., Derbazi, C., Benchora, M.: $\psi$-Caputo fractional differential equations with multi-point boundary conditions by topological degree theory. Res. Nonlinear Anal. 3(4), 167-178 (2020)

10. Tuan, H.T., Mohammadi, H., Rezapour, S.: A mathematical model for COVID-19 transmission by using the Caputo fractional derivative. Chaos Solitons Fractals 140, 110107 (2020). https://doi.org/10.1016/j.chaos.2020.110107

11. Thabet, S.T.M., Etemad, S., Rezapour, S.: On a coupled Caputo conformable system of pantograph problems. Turk. J. Math. 45(1), 496-519 (2021). https://doi.org/10.3906/mat-2010-70

12. Mohammadi, H., Kumar, S., Rezapour, S., Etemad, S.: A theoretical study of the Caputo-Fabrizio fractional modeling for hearing loss due to Mumps virus with optimal control. Chaos Solitons Fractals 144, 110668 (2021). https://doi.org/10.1016/j.chaos.2021.110668

13. Baleanu, D., Jajarmi, A., Mohammadi, H., Rezapour, S.: A new study on the mathematical modelling of human liver with Caputo-Fabrizio fractional derivative. Chaos Solitons Fractals 134, 109705 (2020).

https://doi.org/10.1016/j.chaos.2020.109705 
14. Rezapour, S., Ntouyas, S.K., lqbal, M.Q., Hussain, A., Etemad, S., Tariboon, J.: An analytical survey on the solutions of the generalized double- order $\phi$-integrodifferential equation. J. Funct. Spaces 2021, Article ID 6667757 (2021). https://doi.org/10.1155/2021/6667757

15. Shah, K., Khan, R.A.: Iterative scheme for a coupled system of fractional-order differential equations with three-point boundary conditions. Math. Methods Appl. Sci. 41(3), 1047-1053 (2018). https://doi.org/10.1002/mma.4122

16. Matar, M.M.: Existence of solution for fractional neutral hybrid differential equations with finite delay. Rocky Mt. J. Math. 50(6), 2141-2148 (2020). https://doi.org/10.1216/rmj.2020.50.2141

17. Matar, M.M.: Qualitative properties of solution for hybrid nonlinear fractional differential equations. Afr. Math. 30, 1169-1179 (2019). https://doi.org/10.1007/s13370-019-00710-2

18. Baleanu, D., Etemad, S., Rezapour, S.: On a fractional hybrid integro-differential equation with mixed hybrid integral boundary value conditions by using three operators. Alex. Eng. J. 59(5), 3019-3027 (2020). https://doi.org/10.1016/j.aej.2020.04.053

19. Baleanu, D., Mohammadi, H., Rezapour, S.: Analysis of the model of HIV-1 infection of CD4+ T-cell with a new approach of fractional derivative. Adv. Differ. Equ. 2020, 71 (2020). https://doi.org/10.1186/s13662-020-02544-w

20. Amara, A.: Existence results for hybrid fractional differential equations with three-point boundary conditions. AIMS Math. 5(2), 1074-1088 (2020). https://doi.org/10.3934/math.2020075

21. Tuan, H.T., Ganji, R.M., Jafari, H.: A numerical study of fractional rheological models and fractional Newell-Whitehead-Segel equation with non-local and non-singular kernel. Chinese J. Phys. 68, 308-320 (2020). https://doi.org/10.1016/j.cjph.2020.08.019

22. Matar, M.M.: Approximate controllability of fractional nonlinear hybrid differential systems via resolvent operators J. Math. 2019, Article ID 8603878 (2019). https://doi.org/10.1155/2019/8603878

23. Baleanu, D., Etemad, S., Rezapour, S.: A hybrid Caputo fractional modeling for thermostat with hybrid boundary value conditions. Bound. Value Probl. 2020, 64 (2020). https://doi.org/10.1186/s13661-020-01361-0

24. Granas, A., Dugundji, J.: Fixed Point Theory. Springer, New York (2003)

25. Nikan, O., Jafari, H., Golbabai, A.: Numerical analysis of the fractional evolution model for heat flow in materials with memory. Alex. Eng. J. 59(4), 2627-2637 (2020). https://doi.org/10.1016/j.aej.2020.04.026

26. Ganji, R.M., Jafari, H., Baleanu, D.: A new approach for solving multi variable orders differential equations with Mittag-Leffler kernel. Chaos Solitons Fractals 130, 109405 (2020). https://doi.org/10.1016/j.chaos.2019.109405

27. Abdeljawad, T., Karapinar, E., Pada, S.K., Mlaiki, N.: Solutions of boundary value problems on extended-Branciari b-distance. J. Inequal. Appl. 2020, 103 (2020). https://doi.org/10.1186/s13660-020-02373-1

28. Jarad, F., Alqudah, M.A., Abdeljawad, T.: On more general forms of proportional fractional operators. Open Math. 18(1), 167-176 (2020). https://doi.org/10.1515/math-2020-0014

29. Aksuy, U., Karapinar, E., Erhan, I.M.: Fixed point theorems in complete modular metric spaces and an application to anti-periodic boundary value problems. Filomat 31(17), 5475-5488 (2017). https://doi.org/10.2298/FIL1717475A

30. Lazreg, J.E., Abbas, S., Benchohra, M., Karapinar, E.: Impulsive Caputo-Fabrizio fractional differential equations in b-metric spaces. Open Math. 19(1), 167-176 (2021). https://doi.org/10.1515/math-2021-0040

31. Karapinar, E., Fulga, A., Rashid, M., Shahid, L., Aydi, H.: Large contractions on quasi-metric spaces with an application to nonlinear fractional differential equations. Mathematics 7(5), 444 (2019). https://doi.org/10.3390/math7050444

32. Alqahtani, B., Aydi, H., Karapinar, E., Rakocevic, V.: A solution for Volterra fractional integral equations by hybrid contractions. Mathematics 7(8), 694 (2019). https://doi.org/10.3390/math7080694

33. Abdeljawad, T., Agarwal, R.P., Karapinar, E., Kumari, P.S.: Solutions of the nonlinear integral equation and fractional differential equation using the technique of a fixed point with a numerical experiment in extended $b$-metric space. Symmetry 11(5), 686 (2019). https://doi.org/10.3390/sym11050686

34. Adiguzel, R.S., Aksoy, U., Karapinar, E., Erhan, I.M.: On the solutions of fractional differential equations via Geraghty type hybrid contractions. Appl. Comput. Math. Int. J. 20(2), 313-333 (2021)

35. Baleanu, D., Sajjadi, S.S., Jajarmi, A., Defterli, O., Asad, J.H.: The fractional dynamics of a linear triatomic molecule. Rom. Rep. Phys. 73(1), 105 (2021)

36. Jajarmi, A., Baleanu, D.: On the fractional optimal control problems with a general derivative operator. Asian J. Control 23(2), 1062-1071 (2021). https://doi.org/10.1002/asjc.2282

37. Sajjadi, S.S., Baleanu, D., Jajarmi, A., Pirouz, H.M.: A new adaptive synchronization and hyperchaos control of a biological snap oscillator. Chaos Solitons Fractals 138, 109919 (2020). https://doi.org/10.1016/j.chaos.2020.109919

38. Baleanu, D., Ghanbari, B., Asad, J.H., Jajarmi, A., Pirouz, H.M.: Planar system-masses in an equilateral triangle: numerical study within fractional calculus. Comput. Model. Eng. Sci. 124(3), 953-968 (2020). https://doi.org/10.32604/cmes.2020.010236

39. Jajarmi, A., Baleanu, D.: A new iterative method for the numerical solution of high-order nonlinear fractional boundary value problems. Front. Phys. 8, 220 (2020). https://doi.org/10.3389/fphy.2020.00220

40. Aydogan, S.M., Baleanu, D., Mousalou, A., Rezapour, S.: On high order fractional integro-differential equations including the Caputo-Fabrizio derivative. Bound. Value Probl. 2018, 90 (2018). https://doi.org/10.1186/s13661-018-1008-9

41. Baleanu, D., Mousalou, A., Rezapour, S.: On the existence of solutions for some infinite coefficient-symmetric Caputo-Fabrizio fractional integro-differential equations. Bound. Value Probl. 2017, 145 (2017). https://doi.org/10.1186/s13661-017-0867-9

42. Baleanu, D., Rezapour, S., Saberpour, Z.: On fractional integro-differential inclusions via the extended fractional Caputo-Fabrizio derivation. Bound. Value Probl. 2019, 79 (2019). https://doi.org/10.1186/s13661-019-1194-0

43. Avery, R., Anderson, D., Henderson, J.: Some fixed point theorems of Leggett-Williams type. Rocky Mt. J. Math. 41(2) $371-386(2011)$

44. Devi, A., Kumar, A., Baleanu, D., Khan, A.: On stability analysis and existence of positive solutions for a general non-linear fractional differential equations. Adv. Differ. Equ. 2020, 300 (2020). https://doi.org/10.1186/s13662-020-02729-3

45. Dong, X., Bai, Z., Zhang, W.: Positive solutions for nonlinear eigenvalue problems with conformable fractional differential derivatives. J. Shandong Univ. Sci. Technol. Nat. Sci. 2016(3), 85-91 (2016)

46. Sun, A., Su, Y., Yuan, Q., Li, T.: Existence of solutions to fractional differential equations with fractional-order derivative terms. J. Appl. Anal. Comput. 11(1), 486-520 (2021). https://doi.org/10.11948/20200072 
47. Alsaedi, A., Luca, R., Ahmad, B.: Existence of positive solutions for a system of singular fractional boundary value problems with p-Laplacian operators. Mathematics 8(11), 1890 (2020). https://doi.org/10.3390/math8111890

48. Al-Sadi, W., Zhenyou, H., Alkhazzan, A.: Existence and stability of a positive solution for nonlinear hybrid fractional differential equations with singularity. J. Taibah Univ. Sci. 13(1), 951-960 (2019). https://doi.org/10.1080/16583655.2019.1663783

49. Samko, S.G., Kilbas, A.A., Marichev, O.I.: Fractional Integrals and Derivatives: Theory and Applications. Gordon \& Breach, Philadelphia (1993)

50. Ulam, S.M.: A Collection of Mathematical Problems. Interscience, New York (1960)

51. Hyers, D.H.: On the stability of the linear functional equation. Proc. Natl. Acad. Sci. USA 27(4), 222-224 (1941). https://doi.org/10.1073/pnas.27.4.222

52. Boutiara, A., Etemad, S., Alzabut, J., Hussain, A., Subramanian, M., Rezapour, S.: On a nonlinear sequential four-point fractional $q$-difference equation involving $q$-integral operators in boundary conditions along with stability criteria. Adv. Differ. Equ. 2021, 367 (2021). https://doi.org/10.1186/s13662-021-03525-3

Submit your manuscript to a SpringerOpen ${ }^{\circ}$ journal and benefit from:

- Convenient online submission

$\checkmark$ Rigorous peer review

Open access: articles freely available online

High visibility within the field

- Retaining the copyright to your article

Submit your next manuscript at $\gg$ springeropen.com 